\title{
Network Pharmacology Prediction and Molecular Docking-Based Strategy to Discover the Potential Pharmacological Mechanism of Wen-Yu-Jin against Pulmonary Fibrosis in a Mouse Model
}

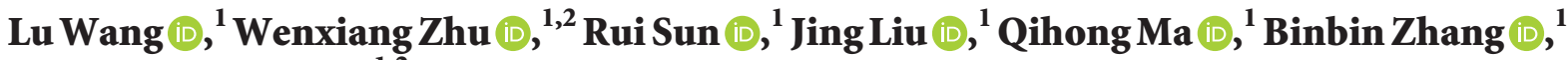 \\ and Yuanyuan Shi $\mathbb{D}^{1,2}$ \\ ${ }^{1}$ School of Life Sciences, Beijing University of Chinese Medicine, Beijing, China \\ ${ }^{2}$ Shenzhen Research Institute, Beijing University of Chinese Medicine, Shenzhen, China \\ Correspondence should be addressed to Yuanyuan Shi; yshi@bucm.edu.cn
}

Received 28 September 2021; Revised 19 January 2022; Accepted 20 January 2022; Published 10 February 2022

Academic Editor: Jorddy Neves Cruz

Copyright (c) $2022 \mathrm{Lu}$ Wang et al. This is an open access article distributed under the Creative Commons Attribution License, which permits unrestricted use, distribution, and reproduction in any medium, provided the original work is properly cited.

\begin{abstract}
Background. Pulmonary fibrosis (PF) is a devastating lung disease, resulting in gas exchange dysfunction until death. The two drugs approved by the FDA, pirfenidone and nintedanib, have obvious side effects. Wen-yu-jin (WYJ), one of the commonly used herbs in China, can treat respiratory diseases. The potential effects and the underlying mechanism of WYJ against PF are unclear. Purpose. Employing network pharmacology, molecular docking, and in vivo and in vitro experiments to explore the potential effects and underlying mechanisms of WYJ in the treatment of PF. Methods. Ultra-high pressure liquid chromatography combined with linear ion trap-orbital tandem mass spectrometry (UHPLC-LTQ-orbital trap) was used to identify compounds of WYJ. We got PF-related targets and WYJ compounds-related targets from public databases and further completed critical targets exploration, network construction, and pathway analysis by network pharmacology. Molecular docking predicted binding activity of WYJ compounds and critical targets. Based on the above results, in vivo and in vitro experiments validated the potential effects and mechanisms of WYJ against PF. Results. 23 major compositions of WYJ were identified based on UHPLC-LTQ-Orbitrap. According to the results of network pharmacology, STAT3, SRC, IL6, MAPK1, AKT1, EGFR, MAPK8, MAPK14, and IL1B are critical therapeutic targets. Molecular docking results showed that most of the compounds have good binding activities with critical targets. The results of in vivo and in vitro experiments showed that WYJ alleviated the process of fibrosis by targeting MAPK and STAT3 pathways. Conclusion. Network pharmacology, molecular docking, and in vivo and in vitro experiments showed the potential effects and mechanisms of WYJ against PF, which provides a theoretical basis for the treatment of WYJ with PF.
\end{abstract}

\section{Introduction}

Pulmonary fibrosis (PF) characterized by progressive dyspnea with respiratory failure [1-3] is an irreversible lung disease [4]. PF susceptibility is closely related to age [5]. Accumulating exposures to numerous risk factors [6], such as smoking, occupational dust, drug stimulation, and bacterial and virus infection, also result in PF. Different risk factors repeatedly promote lung injury, leading to the production of profibrotic cytokines. TGF- $\beta 1$ is one of the most potent profibrosis cytokines $[7,8]$. Profibrotic cytokines further stimulate effector cells activation and migration, leading to the deposition of extracellular matrix (ECM). ECM, a typical feature of pulmonary fibrosis, affects the gas exchange function of the lung. They eventually lead to respiratory failure and death $[9,10]$. Pirfenidone and nintedanib [11], approved by FDA, are the only two effective drugs for clinical PF medical therapies. However, these drugs can only delay the disease's progression and maintain lung function but cannot cure the disease $[12,13]$. Moreover, these drugs can cause adverse effects $[11,13-14]$, resulting in treatment discontinuation and adverse gastrointestinal effects.

Multiple active ingredients and targets of Traditional Chinese Medicines (TCM) arouse the attention of 
pharmacologists. Previous studies showed that TCM or active ingredients from TCM have protective pulmonic [15], representing an attractive source of drug discovery for treating PF. In TCM, there are many theories about the pathogenesis of PF, such as qi stagnation and blood stasis (Qi-Zhi-Xue-Yu in Chinese) and binding of phlegm and qi (Tan-Qi-Hu-Jie in Chinese). Wen-yu-jin (WYJ, Curcuma Radix, 温郁金), derived from the steamed root of Curcuma beauty, has been used for at least 1500 years in China and has the effect of moving qi and blood circulation. Modern pharmacological studies have demonstrated that the ingredients or extracts from WYJ have antibacterial, antitumor anti-inflammatory, and antioxidant effects $[16,17]$. Hui's study showed potential antifibrotic effects of WYJ in liver fibrosis [18]. Therefore, it is necessary to study the therapeutic effects and mechanism of WYJ in the treatment of PF using advanced methods.

In silico approaches have been widely used in the field of drug molecule design, improving efficiency and reducing cost $[19,20]$. Based on bioinformatics, systems biology, and pharmacology, network pharmacology is a promising tool for understanding the complex relationship between drugs and diseases [21]. This tool has been successfully used to reveal the molecular mechanism of TCM in various diseases such as cardiovascular disease [22, 23], diabetes [24], and cancer [25]. Some approaches, such as molecular docking and molecular dynamics simulation (MDS), help researchers understand the pathogenesis of diseases at the molecular level and provide new ideas and theoretical guidance for the design of new drugs $[20,26]$. In a study, researchers organized MDS to understand the resistance mechanism of mutant BCR-ABL protein, which provided ideas for the design of new drugs [27]. Rachel through in silico operations elucidated that Ligand2 has stronger inhibitory potential for cyclin-dependent kinases (CDKs) and can develop into a new selective inhibitor of CDK2 [19].

In our study, we established network pharmacology to explore the molecular mechanism of WYJ against PF. Molecular docking was performed to predict the affinity strength between the active compounds and the critical targets and to explore the antipulmonary fibrosis potential of WYJ. Finally, the antifibrosis mechanism of WYJ was further verified via in vitro and in vivo experiments based on the predicted results.

\section{Materials and Methods}

2.1. Preparation of Chinese Medicine. WYJ was purchased from Tong Ren Tang Co., Ltd. (Beijing, China). The herb was boiled in purified water twice for $1 \mathrm{~h}$ each time. We got lyophilized powder in condensation and freeze-dry of liquids and stored it at $-80^{\circ} \mathrm{C}$ for a series of experiments.

2.2. LC/MS Analysis. We used processed WYJ for in vitro and in vivo experiments. The compounds of processed WYJ were reduced or changed compared with the herbal plant's library. Therefore, we used LC/MS analysis to clarify the compounds of WYJ. The coupling of the UHPLC System
(Thermo Fisher Scientific) equipped with a binary pump, an auto sampler, a column thermostat, and DAD detector, and LTQ-Orbitrap XL (Thermo Fisher Scientific) equipped with an electrostatic ionization source (ESI) was used For LC/MS experiments. Data was controlled and processed by Xcalibur software (Thermo Fisher Scientific). The column used in the LC analysis was Thermo Scientific Hypersil BDS C18 $(2.1 \mathrm{~mm} 150 \mathrm{~mm}, 2.4 \mu \mathrm{m})$. Mobile phase A was water with $0.1 \%$ formic acid while mobile phase B was acetonitrile. The column temperature was set to 35 and the flow rate was $0.3 \mathrm{~mL} / \mathrm{min}$. Elution conditions were summarized as follows: 0-3 min (5\%-5\%B), 3-45 min $\quad(5 \% \sim 75 \% \mathrm{~B}, \quad 45-45.1 \mathrm{~min}$ (75\%-5\%B), and $45.1-50 \mathrm{~min}(5 \%-5 \% \mathrm{~B})$.

The MS analysis was conducted on both the negative and positive ion modes. Flow rates of sheath gas and auxiliary gas were set at $40 \mathrm{Arb}$ and $20 \mathrm{Arb}$, respectively. The capillary voltage was set to $35.0 \mathrm{~V}$. The source temperature was set to 350 and the tube lens was set to $110 \mathrm{~V}$. The source voltage was set to $4 \mathrm{kV}$ and $3 \mathrm{kV}$ in the positive and negative ion modes, respectively.

2.3. Network Pharmacology. According to the compounds identified by UHPLC-LTQ-Orbitrap, we obtained these compound-related targets from the SwissTargetPrediction database [28] (http://www.swisstargetprediction.ch/) and Traditional Chinese Medicine Systems Pharmacology Database and Analysis Platform (TCMSP, https://tcmsp-e.com/). Compounds-related protein names were converted to the gene names using UniProt (https://www.uniprot.org/).

We used "pulmonary fibrosis (PF)," "idiopathic pulmonary fibrosis (IPF)," or "interstitial lung disease (ILD)" as keywords to obtain PF-related targets from GeneCards database v5.0 [29] (https://www.genecards) and OMIM database [30] (https://omim.org/). After combining the targets obtained from different databases using the same keywords, we got the targets of PF by taking the intersection of targets with different keywords.

WYJ-related targets and PF-related targets were intersected to obtain gene symbols. Using the string database (https://www.string-db.org/) [31], the protein-protein interaction (PPI) network was constructed to explore the potential interactions of the intersected gene symbols. Cytoscape v3.6.0 visualized the PPI network. Critical nodes calculated by the median values of three topological features (degree, closeness centrality, and bitterness centrality) were identified as key targets of WYJ.

To determine the biological meaning behind key targets, pathway enrichment was analyzed by Gene Ontology (GO) and the Kyoto Encyclopedia of Genes and Genomes (KEGG) database of David v6.8 (https://david.ncifcrf.gov/) [32, 33]. The threshold value of confident gene enrichment was set at $P<0.05$. Compound-target-pathway network explored the connection of compounds of WYJ, key targets, and important signaling pathways and was visualized using Cytoscape.

2.4. Molecular Docking. The active compounds were molecularly docked with the core proteins based on the results of network pharmacology. The specific operations are as 
TABLE 1: Gene primer sequences.

\begin{tabular}{|c|c|c|c|}
\hline \multicolumn{2}{|c|}{ Gene } & \multirow{2}{*}{ Primer $\left(5^{\prime}-3^{\prime}\right)$} & \multirow{2}{*}{$\begin{array}{l}\text { Accession no. } \\
\text { NM_008084.3 }\end{array}$} \\
\hline \multirow{2}{*}{ Mus-GAPDH } & Forward & & \\
\hline & Reverse & TGGTCCAGGGTTTCTTACTCC & \\
\hline \multirow{2}{*}{ Mus-colla1 } & Forward & CCAAGAAGACATCCCTGAAGTCA & NM_007742.4 \\
\hline & Reverse & TGCACGTCATCGCACACA & \\
\hline \multirow{2}{*}{ Mus-TGF- $\beta 1$} & Forward & ATGACATGAACCGGCCCTT & NM_011577.2 \\
\hline & Reverse & AGTTGGTATCCAGGGCTCTCC & \\
\hline
\end{tabular}

follows: (1) ligands molecule preparation. We downloaded the core compound structures from the Pubchem database (https://pubchem.ncbi.nlm.nih.gov/). All compounds were optimized using the MM2 force field, and saved in.pdbqt format as docking ligands in AutoDock Tools 1.5.6 software (ADT) [34]. (2) Receptor molecule preparation. The crystal structures of proteins were obtained from the RCSB PDB website (http://www.rcsb.org/). Proteins should be human proteins with one or more cocrystallized ligands and crystal structures with small "resolution" value. PyMoL software was utilized to remove water molecules and original ligands [35]. AutoDockTools software was used to add nonpolar hydrogen, calculate the Gasteiger charge, and assign the AD4 type. Receptors were ready and saved in.pdbqt format. (3) Molecular docking. A suitable method of evaluating the reliability of a docking procedure was needed. Prior to docking the ligands against the target proteins, the redocking of the cocrystal structures and their original ligands was carried out. All of the root mean square deviations (RMSDs) were less than $2 \AA$ which indicated the method for docking was reasonable. The grid center of molecular docking was determined by using the cocrystallized ligand in the target protein complex. AutoDock Vina 1.1.2 was utilized for the docking of prepared ligands and proteins. And the spacing and exhaustiveness were set to 0.375 and 8 , respectively. In particular, for IL-6 blind docking was performed with exhaustiveness 24. A Lamarckian genetic algorithm (LGA) was used for the conformational search. The further constraints were set to default for Autodock Vina unless noted. Discovery studio 2019 program was used to visualize the best binding affinity of compounds. The reliability of the applied docking protocol was assessed by redocking cocrystal ligands into the active site of the protein domains. In addition, the positive control drug nintedanib exerts a therapeutic effect on PF. To make the conclusions more objective, we molecularly docked nintedanib with the potential core gene targets we screened.

\subsection{Experimental Verification}

2.5.1. Animals and Experimental Design. Bleomycin (BLM) can induce the formation of a mouse model of $\mathrm{PF}$, and mouse lungs have lung pathological changes similar to those of patients with PF. 75 male mice (18-22 g, 8 weeks old) were purchased from Beijing Vital River Laboratory Animal Technology Co., Ltd. (Beijing, China) and were adaptively reared for one week. After the mice were anesthetized, 15 mice in the control group were given normal saline via the oropharynx, and the rest of the mice were given BLM via the oropharynx to establish the PF model. The dose of BLM was $5 \mathrm{mg} / \mathrm{kg}$. The BLM-induced PF model was randomized into four groups: BLM treatment group (model), BLM with WYJ high concentration treatment group (WYJ-H); BLM with WYJ medium concentration treatment group (WYJ-M), and BLM with WYJ low concentration treatment group (WYJ-L).

According to the previous literature and preliminary experimental results, WYJ-H, WYJ-M, and WYJ-L groups were given WYJ 1.2, 0.6 , and $0.3 \mathrm{~g} / \mathrm{kg}$ doses, respectively. The control group and the model group were treated with normal saline. Treatments were started 12 days after the establishment of the PF model, once a day, for 14 days. We sacrificed all mice on day 26 and stored lung tissue at $-80^{\circ} \mathrm{C}$ for different experiments.

2.5.2. Lung/Body Weight Ratio. We recorded the body weight (BW) and the whole lung weight (PW) after mice were sacrificed. Pulmonary indexes (PI) were calculated using the equation $\mathrm{PI}=\mathrm{PW} / \mathrm{BW} * 100$.

2.5.3. Hydroxyproline Determination (HYP). The lung tissue was processed according to the instructions of the Hydroxyproline Kit (Nanjing Jiancheng Institute of Bioengineering, Nanjing, China). HYP is the main marker of the development of PF. The level of HYP in the lung tissue was measured by the absorbance of $550 \mathrm{~nm}$ and the result was expressed with $\mu \mathrm{g} / \mathrm{lung}$.

2.5.4. Histopathological Examination. Lung tissues fixed with $10 \%$ formalin were embedded in paraffin and sectioned. Sections were stained with hematoxylin and eosin (H\&E) and Masson (Sinopharm Chemical Reagent Beijing Co., Ltd., Beijing, China). Inflammation and collagen deposition of the sections were observed and assessed with an optical microscope.

2.5.5. Culture of Human Pulmonary Fibroblast (HPF). $\mathrm{HPF}$ is a normal human fibroblast cell line from the National Infrastructure of Cell Line Resource (Beijing, China). HPF cells were cultured in medium (DMEM) with $10 \%$ FBS and $1 \% \mathrm{PS}$ and maintained at the cell incubator with $5 \% \mathrm{CO}_{2}$ and $37^{\circ} \mathrm{C}$. Cells were passaged at a confluence of $80 \%-90 \%$ using standard trypsinization techniques. HPF cells stimulated with TGF- $\beta 1(10 \mathrm{ng} / \mathrm{mL})$ were compared with cells that were grown in the same conditions but without stimulation of TGF- $\beta 1$. 


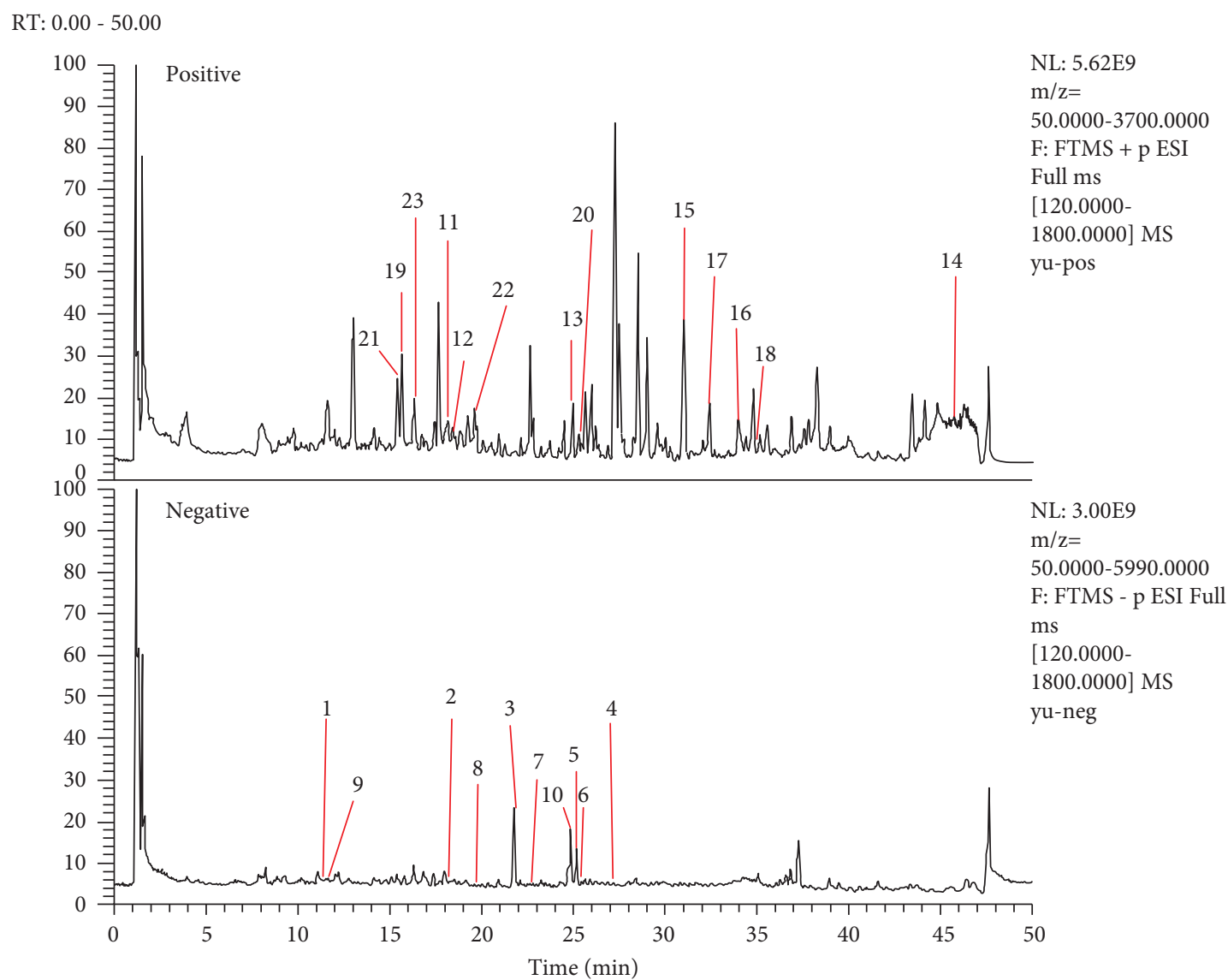

Figure 1: Total ion chromatogram.

TABLE 2: Chemical compounds of WYJ in negative ion mode.

\begin{tabular}{|c|c|c|c|c|c|c|c|c|}
\hline No. & $\begin{array}{c}\mathrm{TR} \\
(\mathrm{min})\end{array}$ & $\begin{array}{c}\text { Molecular } \\
\text { formula }\end{array}$ & $\begin{array}{c}\text { Accurate } \\
\text { mass }[\mathrm{M}-\mathrm{H}]-\end{array}$ & $\begin{array}{c}\text { Calculated } \\
\text { mass }[\mathrm{M}-\mathrm{H}]-\end{array}$ & $\begin{array}{c}\text { Fragmentation } \\
(\mathrm{MS} / \mathrm{MS})\end{array}$ & $\begin{array}{l}\text { Error } \\
(\mathrm{bpm})\end{array}$ & $\begin{array}{c}\text { Identified } \\
\text { compounds }\end{array}$ & PubChem CID \\
\hline 1 & 11.58 & $\mathrm{C} 10 \mathrm{H} 10 \mathrm{O} 3$ & 177.0552 & 177.0555 & $\begin{array}{c}162.0325 ; 113.002 \\
92.9957\end{array}$ & 1.694 & $\begin{array}{c}(\mathrm{Z})-\mathrm{p}- \\
\text { methoxycinnamic } \\
\text { acid }\end{array}$ & 1550936 \\
\hline 2 & 18.13 & $\mathrm{C} 15 \mathrm{H} 18 \mathrm{O} 3$ & 245.1178 & 245.1186 & $\begin{array}{c}\text { 180.9893; } 175.0763 ; \\
57.0345\end{array}$ & 3.264 & Curcolone & 12304272 \\
\hline 3 & 21.68 & $\mathrm{C} 15 \mathrm{H} 18 \mathrm{O} 3$ & 245.1178 & 245.1182 & $\begin{array}{c}224.9971 ; 201.1285 \\
180.9894\end{array}$ & 1.632 & Editors & 46173920 \\
\hline 4 & 27.14 & $\mathrm{C} 15 \mathrm{H} 20 \mathrm{O} 3$ & 247.1334 & 247.1339 & $\begin{array}{c}229.1234 ; 203.1078 \\
189.0921 ; 161.0971\end{array}$ & 2.023 & Curcumenolactone A & 10083354 \\
\hline 5 & 25.28 & $\mathrm{C} 15 \mathrm{H} 22 \mathrm{O} 3$ & 249.1491 & 249.1497 & $\begin{array}{l}228.9897 ; 205.1598 \\
154.9738 ; 112.9857\end{array}$ & 2.408 & Procurcumadiol & 14633012 \\
\hline 6 & 25.29 & $\mathrm{C} 15 \mathrm{H} 24 \mathrm{O} 3$ & 251.1647 & 251.1654 & $\begin{array}{c}207.1753 ; 191.1442 \\
125.0972 ; 57.0346\end{array}$ & 2.787 & Zedoarondiol & 24834047 \\
\hline 7 & 22.62 & $\mathrm{C} 15 \mathrm{H} 22 \mathrm{O} 4$ & 265.144 & 265.1445 & $\begin{array}{c}194.9886 ; 112.9856 \\
68.9957\end{array}$ & 1.886 & Zedoalactone A & 15226639 \\
\hline 8 & 19.77 & $\mathrm{C} 15 \mathrm{H} 12 \mathrm{O} 5$ & 271.0607 & 271.0613 & $\begin{array}{c}\text { 186.9991; } 151.0036 \\
119.0501\end{array}$ & 2.214 & Naringenin & 439246 \\
\hline 9 & 11.56 & $\mathrm{C} 15 \mathrm{H} 20 \mathrm{O} 5$ & 279.1233 & 279.1236 & $\begin{array}{c}235.1339 ; 217.1232 \\
181.0869 ; 137.097\end{array}$ & 1.075 & Zedoalactone B & 15226640 \\
\hline 10 & 24.88 & $\mathrm{C} 21 \mathrm{H} 22 \mathrm{O} 6$ & 369.1338 & 369.1347 & $\begin{array}{l}354.1110 ; 247.0974 \\
218.0585 ; 140.0114\end{array}$ & 2.438 & 1,2-Dihydrocurcumin & 5372374 \\
\hline
\end{tabular}


TABLE 3: Chemical compounds of WYJ in negative ion mode.

\begin{tabular}{|c|c|c|c|c|c|c|c|c|}
\hline No. & $\begin{array}{c}\mathrm{TR} \\
(\mathrm{min})\end{array}$ & $\begin{array}{l}\text { Molecular } \\
\text { formula }\end{array}$ & $\begin{array}{c}\text { Accurate } \\
\text { mass } \\
{[\mathrm{M}+\mathrm{H}]+}\end{array}$ & $\begin{array}{l}\text { Calculated mass } \\
\qquad[\mathrm{M}+\mathrm{H}]+\end{array}$ & $\begin{array}{l}\text { Fragmentation } \\
\text { (MS/MS) }\end{array}$ & $\begin{array}{c}\text { Error } \\
\text { (bpm) }\end{array}$ & $\begin{array}{l}\text { Identified } \\
\text { compounds }\end{array}$ & PubChem CID \\
\hline 11 & 17.67 & $\mathrm{C} 10 \mathrm{H} 12 \mathrm{O}$ & 149.0966 & 149.0962 & 121.0649 & -2.683 & Cuminal & 326 \\
\hline 12 & 18.02 & $\mathrm{C} 19 \mathrm{H} 16 \mathrm{O} 4$ & 309.1127 & 309.1111 & $281.0920 ; 62.9826$ & -5.176 & Bisdemethoxycurcumin & 45934475 \\
\hline 13 & 24.07 & $\mathrm{C} 15 \mathrm{H} 20$ & 201.1643 & 201.1638 & $\begin{array}{c}169.0860 ; 141.091 \\
113.0601\end{array}$ & -2.486 & Calacorene & 12302243 \\
\hline 14 & 45.72 & $\mathrm{C} 15 \mathrm{H} 22$ & 203.18 & 203.1796 & $\begin{array}{c}175.1482 ; 133.1013 \\
109.1016 ; 81.0706\end{array}$ & -1.969 & $(+)$-alpha-curcumene & 3083834 \\
\hline 15 & 30.43 & $\mathrm{C} 15 \mathrm{H} 16 \mathrm{O}$ & 213.1279 & 213.1275 & $195.1169 ; 157.1013$ & -1.877 & Pyrocurzerenone & 12314812 \\
\hline 16 & 32.49 & $\mathrm{C} 15 \mathrm{H} 20 \mathrm{O}$ & 217.1592 & 217.1588 & $\begin{array}{c}\text { 161.0961; 137.0961; } \\
95.0860\end{array}$ & -1.842 & Ar-turmerone & 160512 \\
\hline 17 & 31 & $\mathrm{C} 15 \mathrm{H} 16 \mathrm{O} 2$ & 229.1228 & 229.1223 & NA & -2.182 & Gweicurculactone & 130117 \\
\hline 18 & 34.07 & $\mathrm{C} 15 \mathrm{H} 18 \mathrm{O} 2$ & 231.1385 & 231.1381 & $\begin{array}{c}213.1275 ; 173.0962 \\
85.0653\end{array}$ & -1.731 & Epicurzerenone & 5317062 \\
\hline 19 & 15.7 & $\mathrm{C} 15 \mathrm{H} 20 \mathrm{O} 2$ & 233.1541 & 233.1536 & $\begin{array}{c}215.1432 ; 187.1482 ; \\
175.1118\end{array}$ & -2.145 & Furanogermenone & 6439596 \\
\hline 20 & 24.49 & $\mathrm{C} 15 \mathrm{H} 20 \mathrm{O} 2$ & 233.1541 & 233.154 & NA & -0.429 & Turnaround A & 15858385 \\
\hline 21 & 15.39 & $\mathrm{C} 15 \mathrm{H} 22 \mathrm{O} 2$ & 235.1698 & 235.1692 & $\begin{array}{c}217.1587 ; 189.1638 ; \\
135.1169 ; 97.0653\end{array}$ & -2.551 & Germacrone-13-al & 46173921 \\
\hline 22 & 19.31 & $\mathrm{C} 15 \mathrm{H} 24 \mathrm{O} 2$ & 237.1854 & 237.1847 & $\begin{array}{c}219.1381 ; 173.1362 \\
133.1013\end{array}$ & -2.951 & Circumstance & 15071433 \\
\hline 23 & 16.39 & $\mathrm{C} 15 \mathrm{H} 20 \mathrm{O} 3$ & 249.149 & 249.1485 & 231.1379 & -2.007 & Curcumenolactone B & 10264037 \\
\hline
\end{tabular}

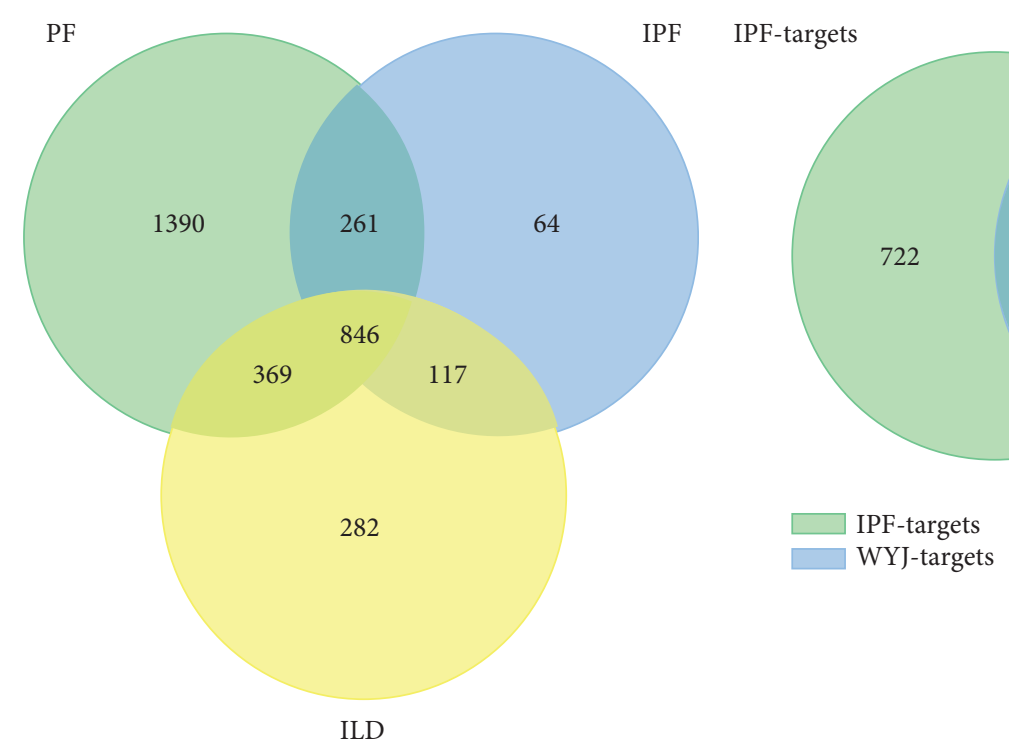

(a)

(b)

Figure 2: (a) Venn diagram of IPF-related targets. (b) Venn diagram of potential targets for WYJ treatment of PF.

2.5.6. Cell Viability Analysis. We assessed the viability of HPF cells pretreated with WYJ using Cell Counting Kit-8 (CCK-8, CK001, Lablead). HPF cells were seeded into a 96well plate in the density of $2 \times 10^{4}$ cells/well and treated with or without WYJ $(0,0.1,1,10,20$, or $50 \mu \mathrm{g} / \mathrm{mL})$. In each well, $200 \mu \mathrm{L}$ media was added with $10 \%$ CCK- 8 solution after $48 \mathrm{~h}$ and then incubated at 37 for 1-2h. The absorbance of the well was measured at $450 \mathrm{~nm}$ using a microplate reader (Molecular Devices, Sunnyvale, CA, USA).

2.5.7. Quantitative Real-Time Polymerase Chain Reaction ( $q R T-P C R)$. Total RNA extracted from HPF cells or mouse lung tissues using Trizol reagent (Qiagen, New York, USA) 


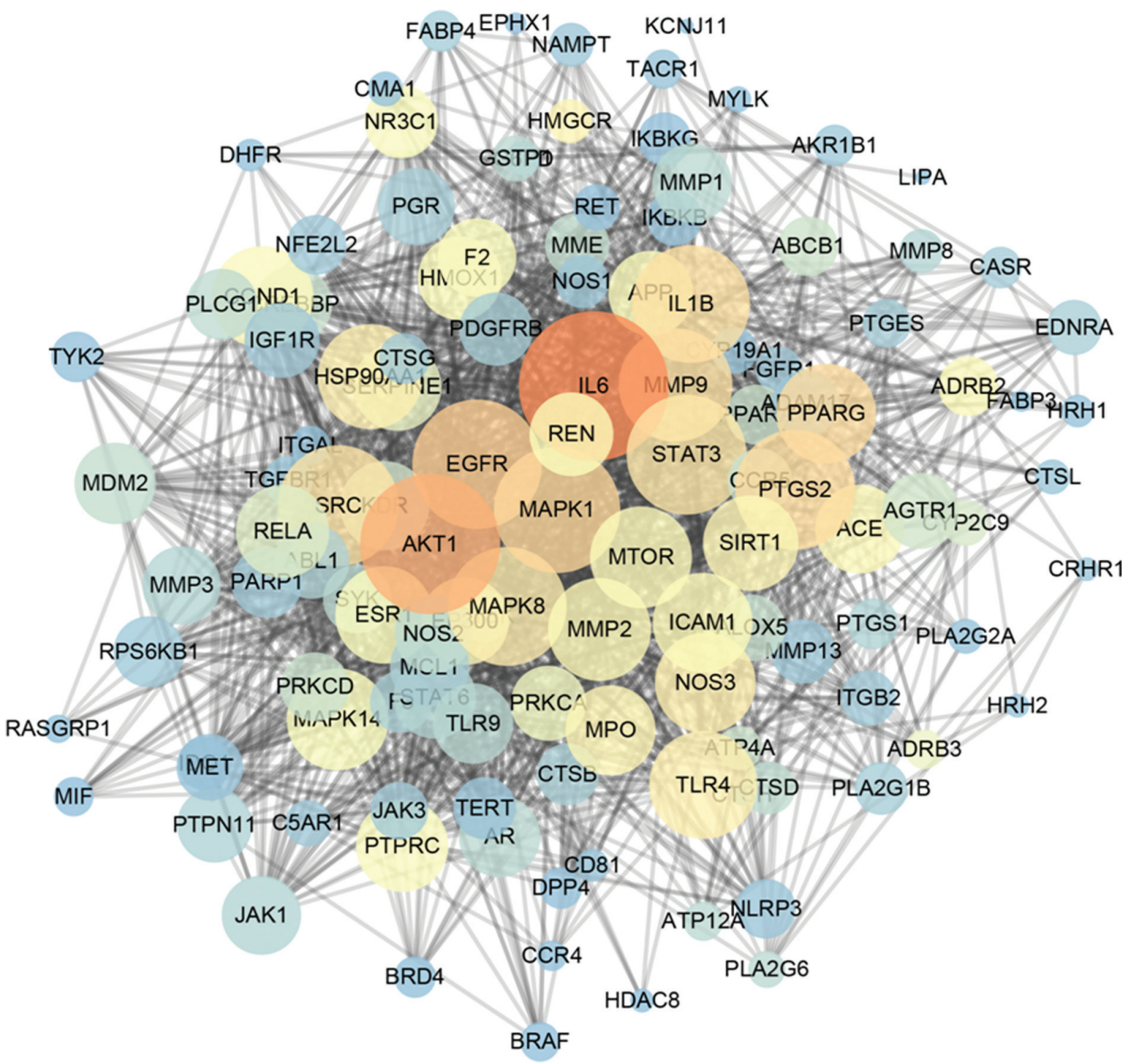

FIGURE 3: PPI network of potential targets.

was purified with RNeasy column (Qiagen, New York, USA). The cDNA was obtained from purified RNA following the manufacturer's instructions of a reverse transcription kit (Qiagen, New York, USA). Full using QuantStudio6 Flex, gene expression analysis was performed following instructions of QuantiFast SYBR Green PCR kit (Qiagen, New York, USA). Fold changes of relative gene expression were calculated using the $2^{-\triangle \triangle \mathrm{Ct}}$ method. Gene primer sequences are shown in Table 1.

2.5.8. Western Blotting. Proteins obtained from lung tissue or cells were further analyzed with Bicinchoninic Acid (Applygen, Beijing, China) for protein concentration. Protein at 30-50 $\mu \mathrm{g} /$ lanes was loaded and separated by SDS-PAGE gel electrophoresis. After SDS-PAGE, proteins were transferred to the polyvinylidene fluoride (PVDF) membranes (Millipore, Massachusetts, USA). Membranes were blocked with 5\% fatfree milk dissolved with Tris-buffered saline with Tween 20 (TBST) for $120 \mathrm{~min}$ at room temperature. Rinsed with TBST, these membranes were incubated with primary antibodies at $4^{\circ} \mathrm{C}$ overnight. On the second day, blots were rinsed with TBST and then incubated with secondary antibody for $1 \mathrm{~h}$. Blots were visualized by gel imager. GAPDH was used for loading positive control and the results were analyzed using Image J.

Antibodies: anti- $\alpha$-SMA (ab5694, abeam, Cambridge, UK), anti-Collagen 1 (14695, Proteintech), anti-p-MAPK (ab201015, abeam), anti-MAPK (ab184699, abeam), anti-pSTAT3 (ab76315, abeam), anti-STAT3 (ab68153, abeam), anti-GAPDH (60004, Proteintech, Chicago, USA), anti- 


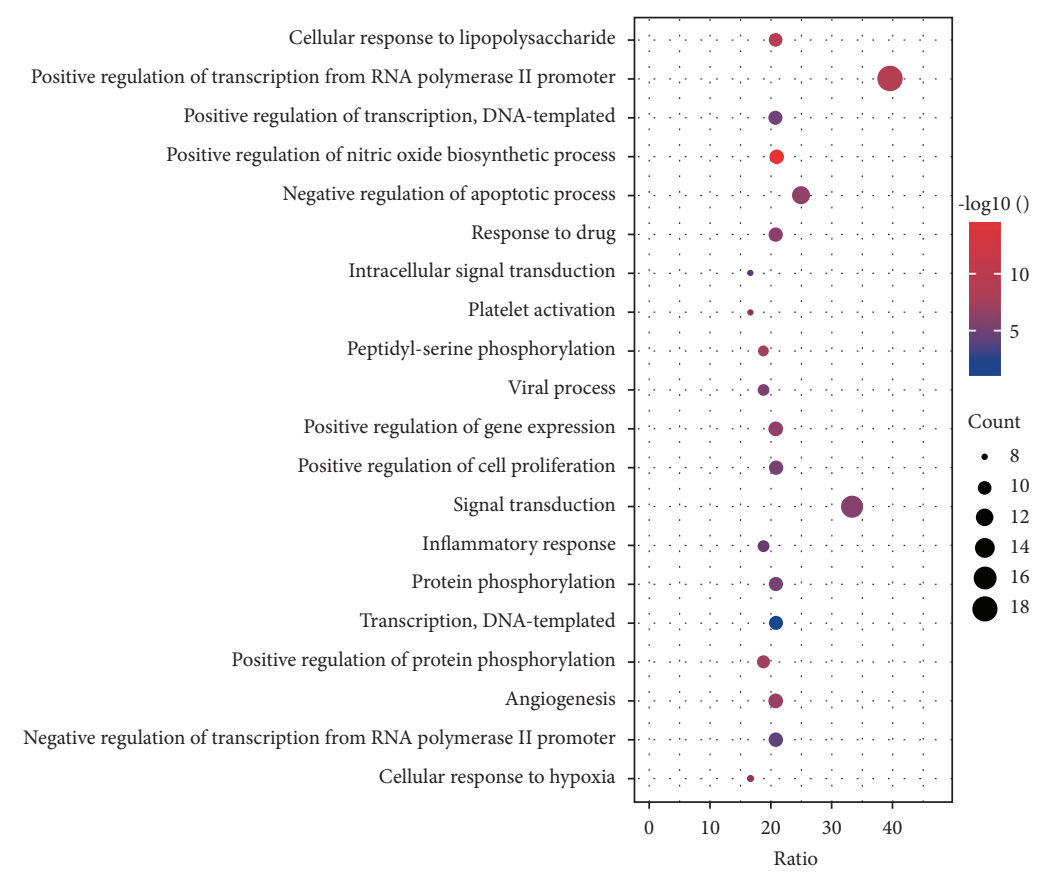

(a)

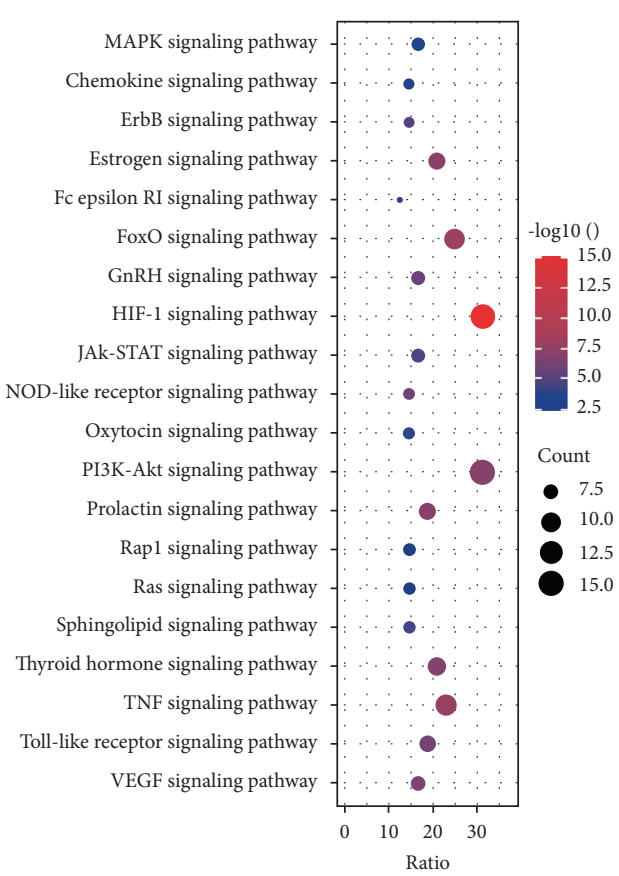

(b)

FIGURE 4: Bubble diagram of functional analysis. (a) Enrichment analysis of a biological process. (b) KEGG pathway enrichment analysis.

rabbit IgG (ab205718, abeam), and anti-mouse IgG (ab6728, abeam).

2.5.9. Data and Statistical Analysis. One-way ANOVA was performed using GraphPad Prism software (version 8.0.2). $P$ values $<0.05$ are adopted as statistically significant.

\section{Results}

3.1. Identified Ingredients of WYJ. Using UHPLC-LTQOrbitrap to detect and identify chemical compounds of WYJ (Figure 1), a total of 23 compounds were confirmed based on comprising with the published data. Information about the identified compounds is summarized in Tables 2 and 3.

3.2. Network and Pathway Analysis. Based on the network pharmacology methods above, $436 \mathrm{WYJ}$-related targets were obtained from SwissTargetPrediction and TCMSP after removing duplicate items. Information about WYJ-related targets is provided in Supplementary Table S1. Combining the results from GeneCards and OMIM database, a total of 2,867 targets for PF, 1,287 targets for IPF, and 1,613 targets for ILD were collected. As shown in Figure 2(a), 846 overlapping targets were critical targets for PF. The details are shown in Supplementary Table S2. Combining 463 WYJrelated targets with 846 PF-related targets, 124 targets could be potential targets for the WYJ treatment of PF
(Figure 2(b)). Detailed information about 124 potential targets is provided in Supplementary Table S3.

STRING databases were used to explore the underlying interactions of 124 potential targets. There were 124 nodes and 1716 edges of the PPI network visualized by Cytoscape (Figure 3). According to the conditions of three topological (degree $>22$, bitterness centrality $>0.00208$, and, closeness centrality $>0.5371179$ ), 48 nodes were identified as the key targets of WYJ against PF (Supplementary Table S4).

48 key targets obtained from PPI were selected to investigate the biological processes $(\mathrm{BP})$ and mechanisms of WYJ on PF treatment. Results showed that BP were involved in PF treatment (Figure 4(a)), including positive regulation of transcription from RNA polymerase II promoter, inflammatory response, and signal transduction, etc. Concerning prediction of KEGG by David 6.8, the results revealed that most of the therapeutic targets are associated with signal transduction (e.g., MAPK signaling pathway and PI3K-protein kinase B (Akt) signaling pathway), inflammation (e.g., TNF signaling pathway and HIF-1 signaling pathway), and immune response (e.g., toll-like receptor signaling pathway and Jak-STAT signaling pathway). 20 significant KEGG pathway terms are shown in Figure 4(b).

Compound-target-pathway network explored the connection of compounds of WYJ, key targets, and 20 pathways. The integrated compound-target-pathway network comprised 69 nodes and 258 edges (Figure 5). STAT3, SRC, IL6, MAPK1, AKT1, EGFR, MAPK8, MAPK14, and IL1B were 


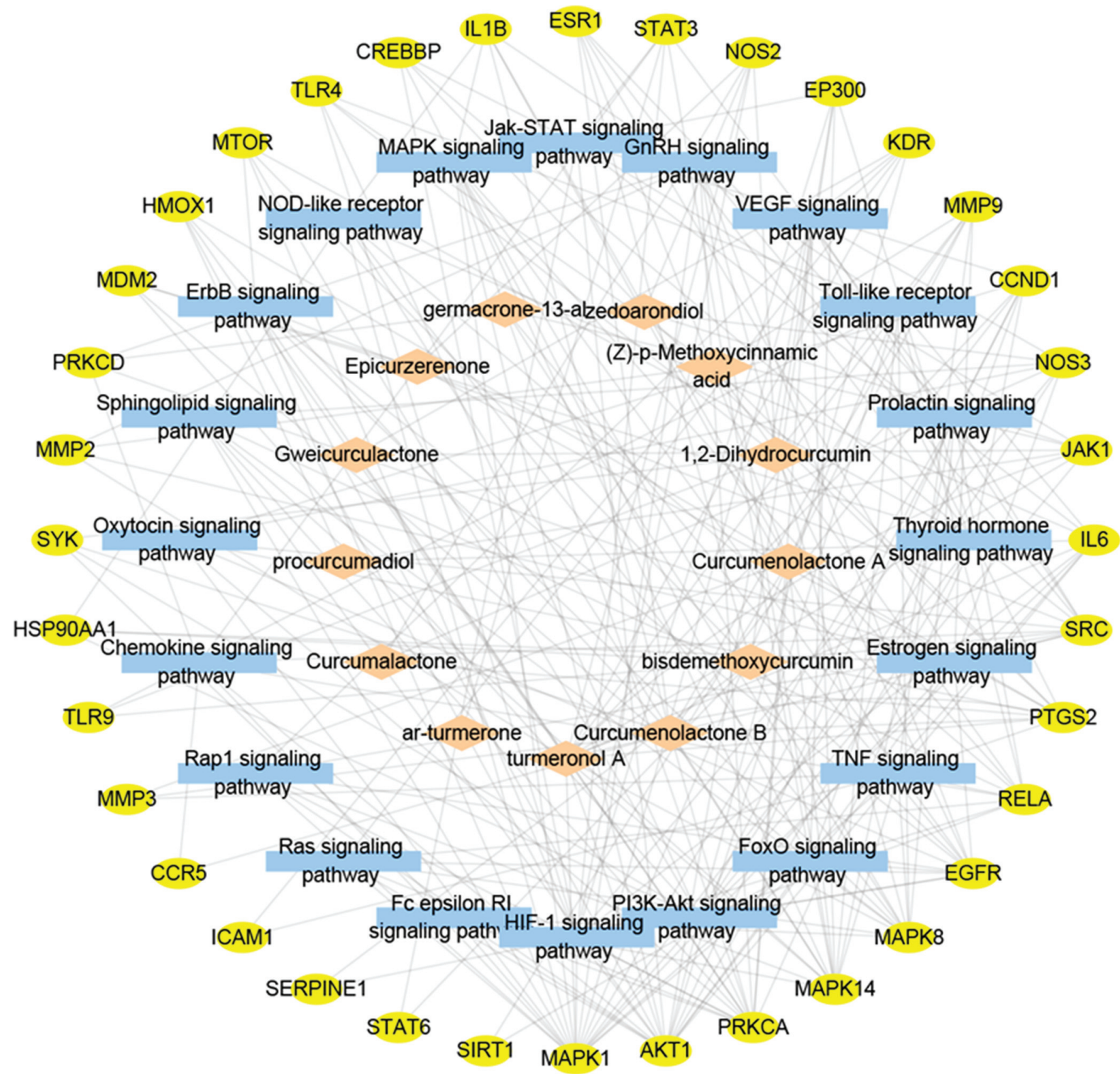

FIgURE 5: The integrated compound-target-pathway network visualized by Cytoscape.

the nine most important nodes in the compound-targetpathway network comparing bitterness centrality, closeness centrality, and degree (Supplementary Table S5). 1,2Dihydrocurcumin, ar-turmerone, bisdemethoxycurcumin, Curcumalactone, Curcumenolactone A, Curcumenolactone B, Epicurzerenone, germacrone-13-al, Gweicurculactone, (Z)-p-methoxycinnamic acid, procurcumadiol, turmeronol $\mathrm{A}$, and zedoarondiol were the critical compounds.

3.3. Molecular Docking. RMSDs between the cocrystal structures and their original ligands were less than $2 \AA$, which indicated the method for docking was reasonable (Supplementary-Molecular Docking). We found that the affinity energies of most small molecules docking with proteins were less than $-5.0 \mathrm{kcal} / \mathrm{mol}$ (SupplementaryMolecular Docking). Heat map of molecular docking is shown in the Figure 6(a). The major binding interactions between the compounds and target proteins include hydrophobic interaction and hydrogen bonding. For example (Figure 6(b)), bisdemethoxycurcumin formed four hydrogen bonds with amino acid residues Glu71, Met109, Gly110, and Asp112 with distances of $1.76 \AA$, $1.93 \AA, 2.64 \AA$, and $1.72 \AA$, respectively, and formed hydrophobic interactions with amino acid residues Lys53, Ile84, Ala111, and Leu167.

\subsection{Experimental Verification of WYJ Effects on the Pulmonary Fibrosis}

3.4.1. WYJ Alleviated BLM-Induced Pulmonary Fibrosis in Mice. The BLM-induced PF model was used to verify the mechanism of WYJ on the treatment of PF. We observed significant decreases in survival rate, severe weight loss, and 


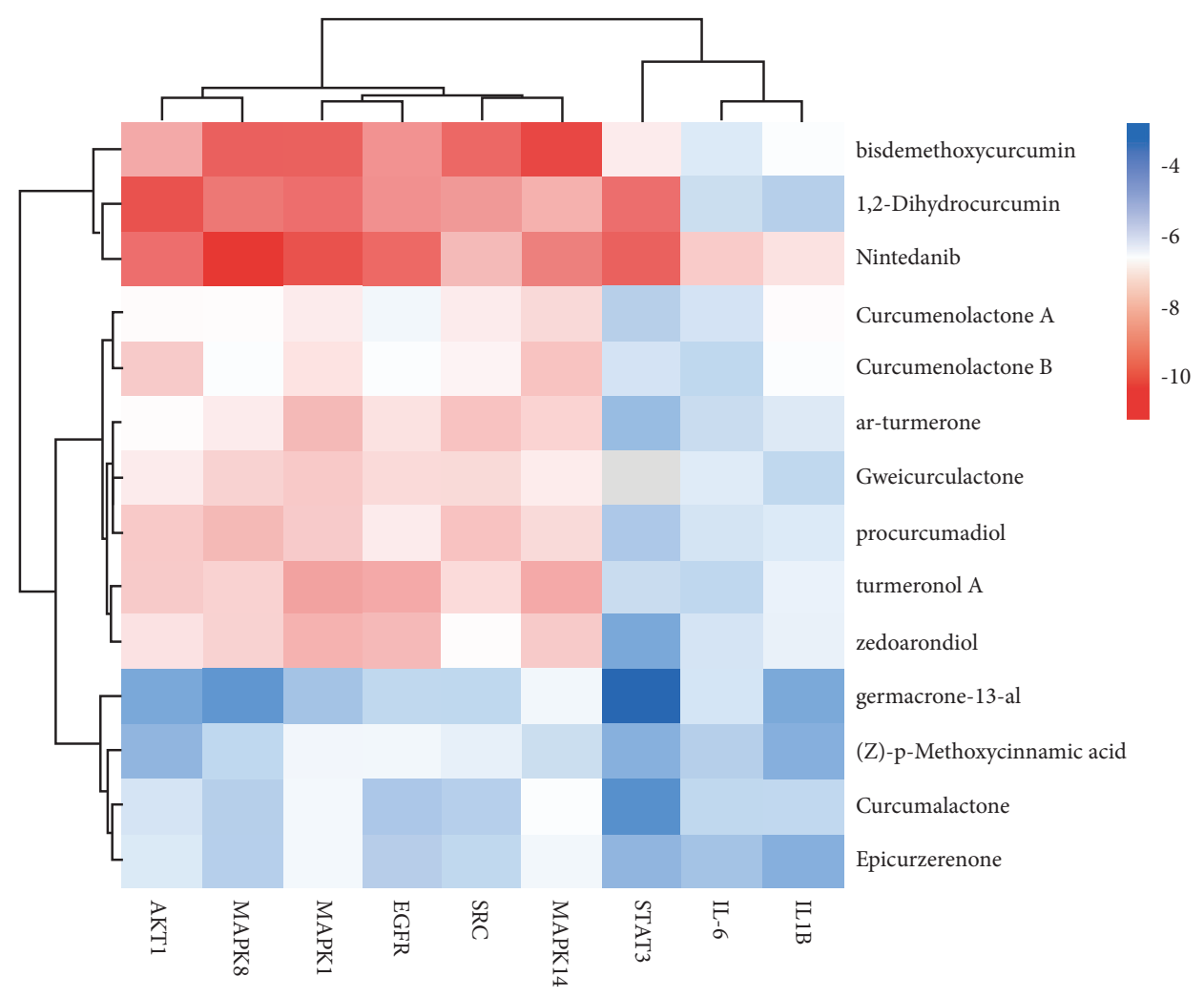

(a)

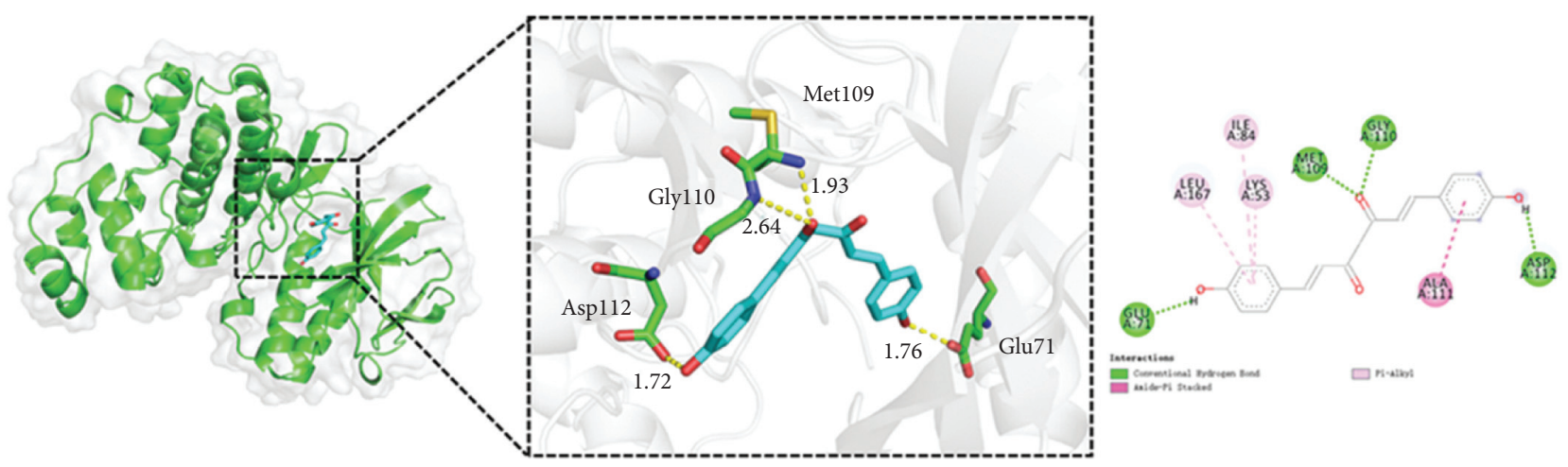

(b)

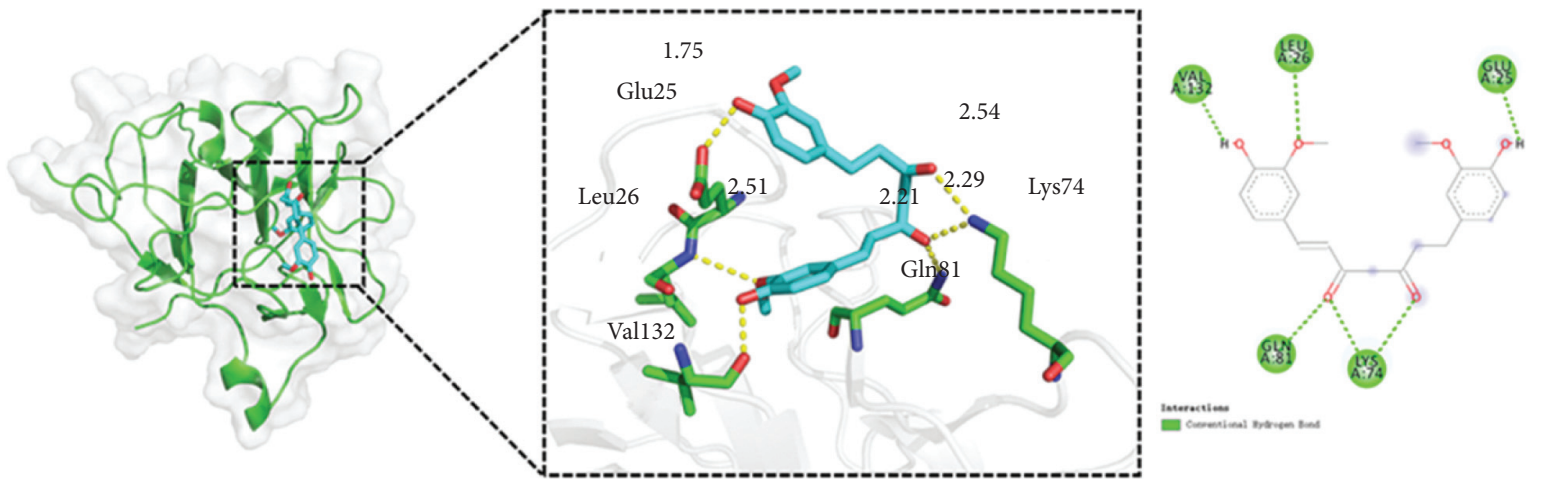

(c)

FIgURE 6: Continued. 

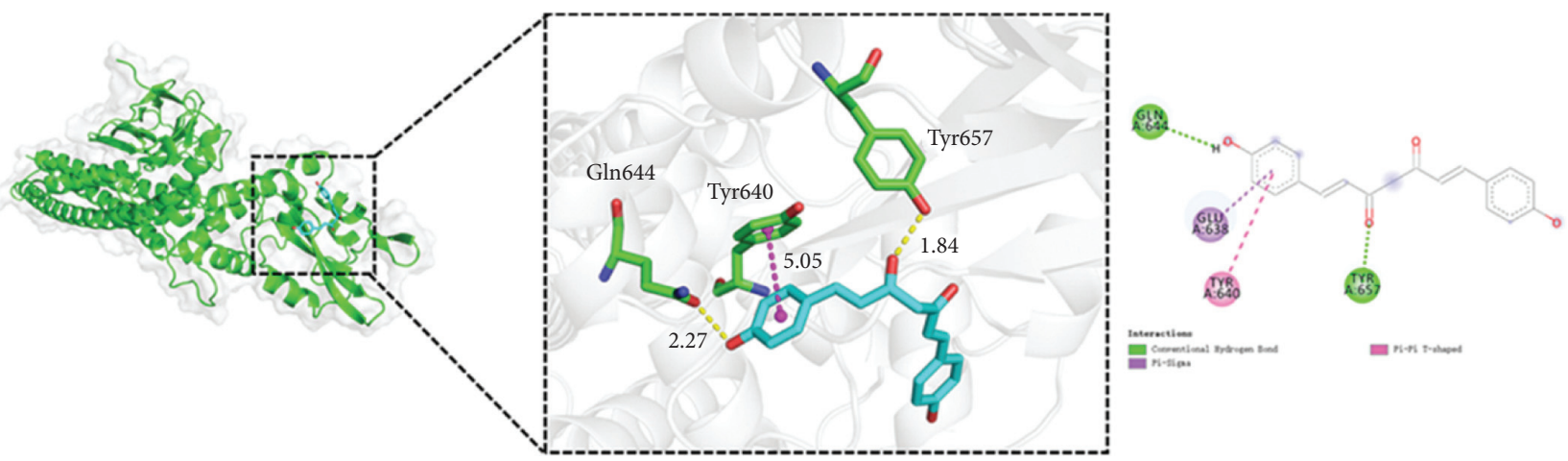

(d)
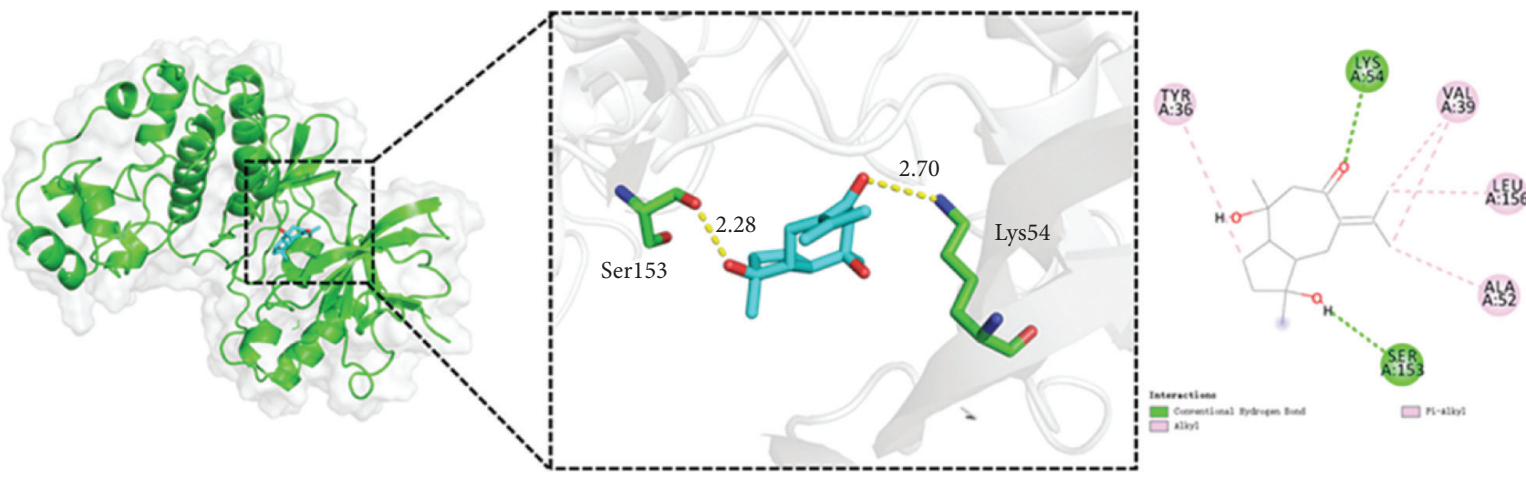

(e)

Figure 6: (a) Heat map of molecular docking. The redder the area, the lower the binding energy and the more stable the docking result. (b) Schematic diagram of the interplay between MAPK14 and bisdemethoxycurcumin. (c) Schematic diagram of the interplay between IL1B and 1,2-dihydrocurcumin. (d) Schematic diagram of the interplay between STAT3 and bisdemethoxycurcumin. (e) Schematic diagram of the interplay between MAPK1 and zedoarondiol.

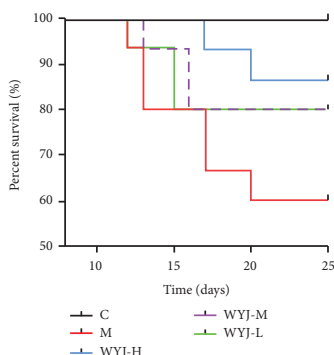

(a)
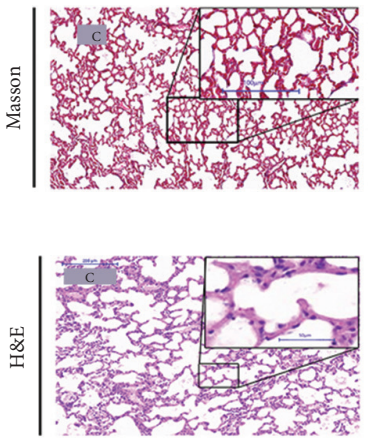

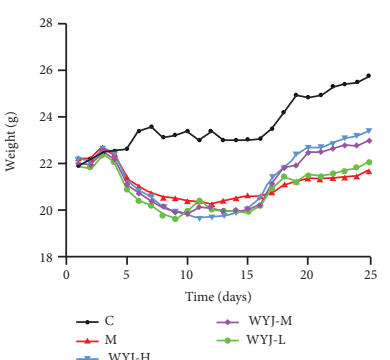

(b)

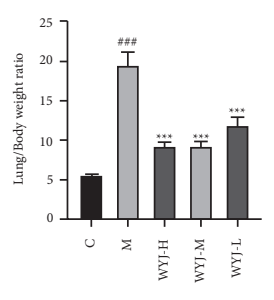

(c)
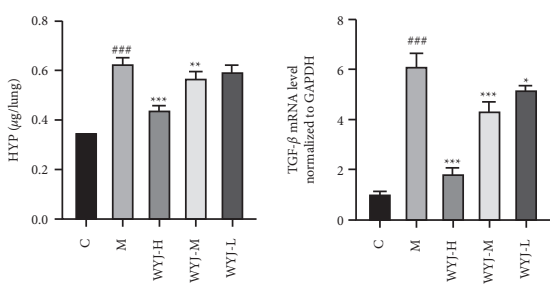

(d)

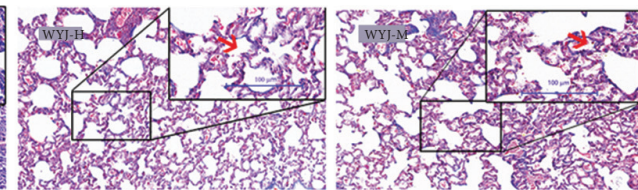

(f)
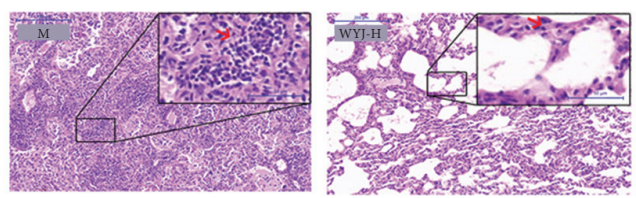
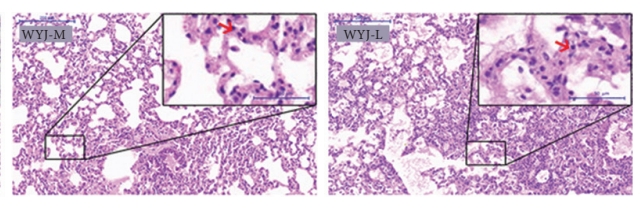

(e)

(g)

Figure 7: WYJ alleviated BLM-induced pulmonary fibrosis in mice. (a) Survival rate. (b) The body weight. (c) Lung/body weight ratio. (d) Hydroxyproline. (e) Lung tissue TGF- $\beta 1$ mRNA. (f) The representative images of Masson staining. (g) The representative images of H\&E staining. $\mathrm{C}$, the control group. $\mathrm{M}$, the model group. The datum was presented as meaning $\pm \operatorname{SEM}(n \geq 3)$. ${ }^{*} P<0.05,{ }^{* *} P<0.01$ and ${ }^{* * *} P<0.001$ versus the model group; ${ }^{\#} P<0.05,{ }^{\# \#} P<0.01$ and ${ }^{\# \# \#} P<0.001$ versus the control group. 


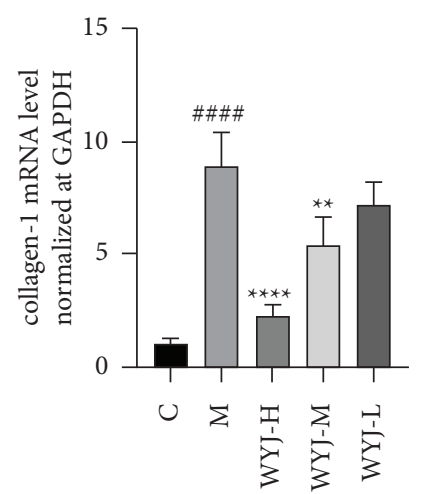

(a)
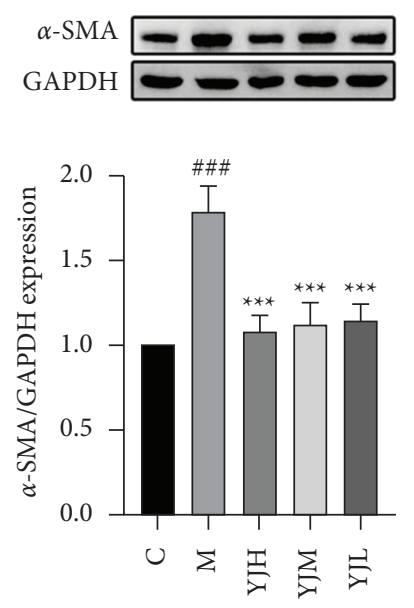

(b)
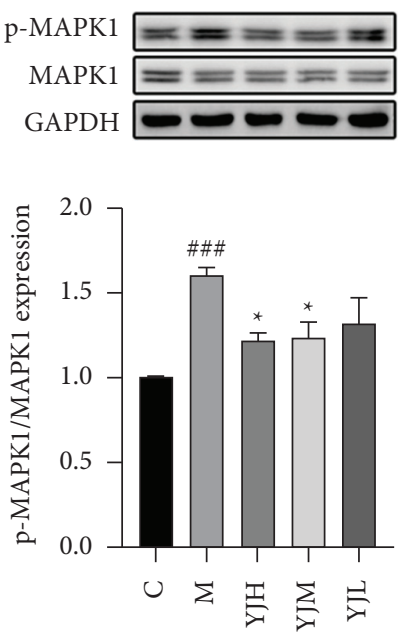

(c)
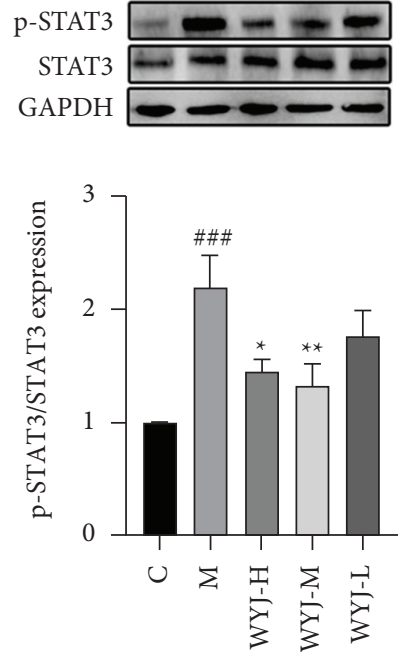

(d)

FIGURE 8: The effect of WYJ on BLM-induced pulmonary fibrosis mice. (a) mRNA expression of collagen I in lung tissues was measured by qRTPCR. (b-d) The relative protein levels of $\alpha$-SMA, p-MAPK1, and p-STAT3. C, the control group. M, the model group. The datum was presented as meaning $\pm \operatorname{SEM}(n \geq 3) .{ }^{*} P<0.05,{ }^{* *} P<0.01$ and ${ }^{* * *} P<0.001$ versus the model group; ${ }^{\#} P<0.05,{ }^{\# \#} P<0.01$ and ${ }^{\# \# \#} P<0.001$ versus the control group.

increase lung/body weight ratio in the BLM-induced PF model compared with the control group. The WYJ treatment increased the survival rate, attenuated weight loss, and reduced the lung/body weight ratio (Figures $7(\mathrm{a})-7(\mathrm{c})$ ). HYP, an important fibrosis indicator of collagen deposition, increased in the model group and decreased by the WYJ treatment (Figure $7(\mathrm{~d})$ ). A large amount of TGF- $\beta 1$ was observed during the development of PF [34, 35]. In our study, WYJ treatment downregulated the expression of TGF- $\beta 1$ mRNA in lung tissue of the PF model (Figure 7(e)). As revealed by Masson staining (Figure $7(\mathrm{f})$ ) and $\mathrm{H} \& \mathrm{E}$ staining (Figure $7(\mathrm{~g})$ ), WYJ treatment decreased the infiltration of inflammatory cells and relieved thrombus and structure destruction compared with the model group. The above results confirmed that WYJ exerted a therapeutic effect on PF.

Excessive deposition of ECM, such as collagen I [36], is characteristic of fibrosis. Compared with the model group, the WYJ treatment suppressed the mRNA expression of collagen I (Figure 8(a)). The differentiation of lung fibroblasts into myofibroblasts is essential for the development of PF $[37,38]$, and $\alpha$-SMA is a marker of myofibroblasts [35]. The WB results indicated that the expression of $\alpha$-SMA decreased after WYJ treatment compared with the model group (Figure $8(\mathrm{~b})$ ), indicating that WYJ reduced the production of myofibroblasts.

According to the results of network pharmacology analysis, it is worth noting that STAT3 is the most important target in the compound-target-pathway network. Moreover, IL6, MAPK1, AKT1, EGFR, MAPK8, MAPK14, and IL1B were the key targets interacting closely with the MAPK signaling pathway. Therefore, we speculated that WYJ might improve PF mainly via the STAT3 and MAPK signaling pathways. Abundant evidence has indicated that STAT3 plays a key role in the occurrence and development of fibrosis $[39,40]$, whereas the conduction of the MAPK signaling pathway is closely related to PF [41, 42]. Therefore, we experimentally verified the expression of key proteins in these two pathways by WB. Compared with the control group, the phosphorylation levels of MAPK1 increased, while this trend was markedly reduced after WYJ treatment (Figure 8(c)). In addition, the same manner was observed in the expression level of phosphorylated STAT3 (Figure $8(\mathrm{~d})$ ). Those results revealed that the protective effect of WYJ on PF might be related to the inhibition of MAPK and STAT3 pathways.

\subsubsection{WYJ Inhibited Fibroblast Activation in In Vitro} Experiments. In vitro experiments further verified the antipulmonary fibrosis effect of WYJ. We observed no change in cell morphology at different concentrations of WYJ using microscopy. However, CCK-8 results showed that, at a concentration of $50 \mu \mathrm{g} / \mathrm{mL}$, the viability of HPF cells decreased slightly (Figures 9(a) and 9(b)). Therefore, we used $0.1-20 \mu \mathrm{g} / \mathrm{mLWYJ}$ to observe the effect of WYJ on TGF-induced HPF cells.

Activated lung fibroblasts, the primary roles in the progress of fibrogenesis, express excessive $\alpha$-SMA and collagen I. We used TGF- $\beta 1$ to promote fibroblast activation [35] and evaluated the expression of $\alpha$-SMA and collagen I after WYJ treatment. The results demonstrated that HPF cells stimulated with TGF- $\beta 1$ significantly increased the production of $\alpha$-SMA and collagen I protein. HPF cells pretreated with WYJ markedly decreased expression of $\alpha$-SMA and collagen I protein (Figures 9(c) and 9(d)). And the results also showed that WYJ prevented the activation of fibroblasts by downregulating p-MAPK1 and p-STAT3 in vitro (Figures $9(\mathrm{e})$ and $9(\mathrm{f})$ ). 


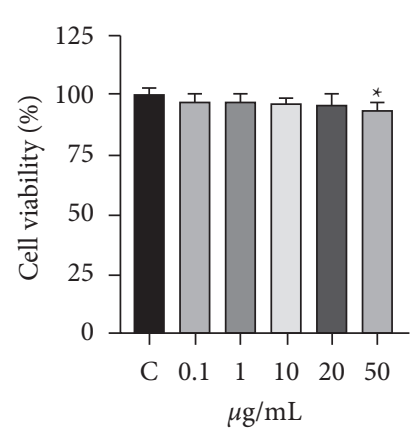

(a)
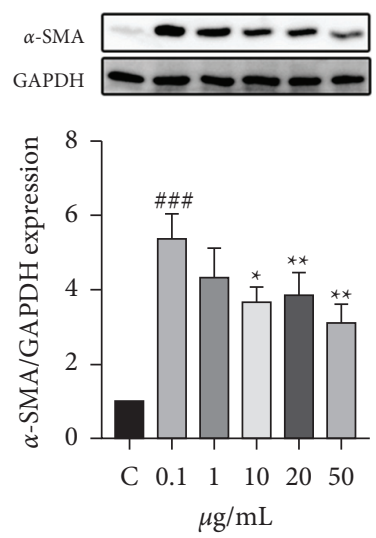

(c)
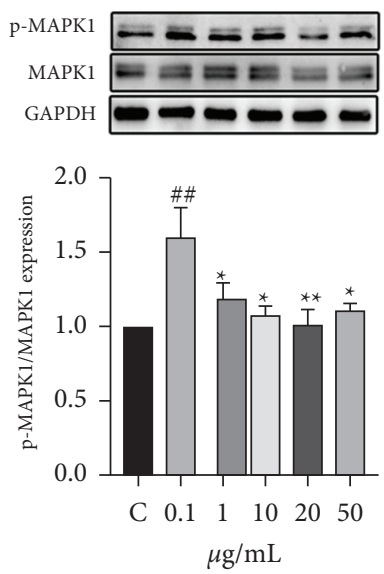

(e)

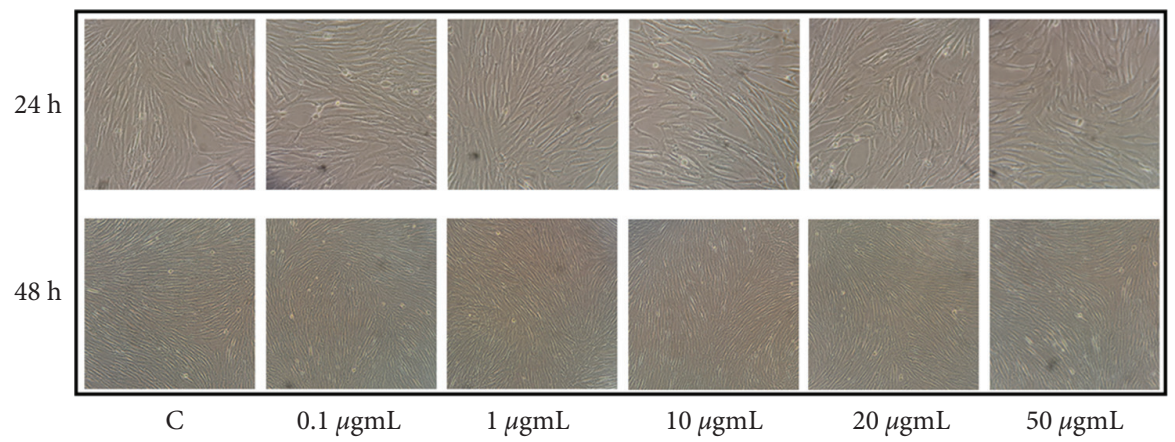

(b)
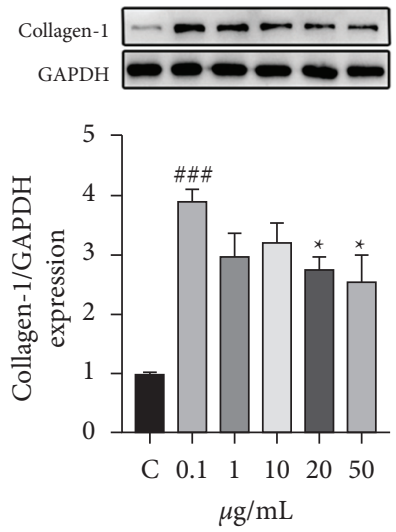

(d)
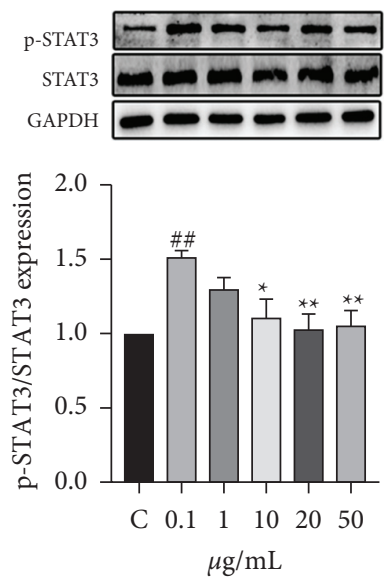

(f)

Figure 9: (a, b) The effect of WYJ on HPF activity. (c-f) The relative protein levels of $\alpha$-SMA, collagen I, p-MAPK1, and p-STAT3. C, the control group. $\mathrm{M}$, the model group. The datum was presented as meaning \pm SEM $(n \geq 3) .{ }^{*} P<0.05,{ }^{* *} P<0.01$ and ${ }^{* * *} P<0.001$ versus the model group; ${ }^{\#} P<0.05,{ }^{\# \#} P<0.01$, and ${ }^{\# \# \#} P<0.001$ versus the control group.

\section{Discussion and Conclusions}

$\mathrm{PF}$ is a chronic progressive tissue repair response that can lead to irreversible scarring and remodeling of the lungs [9]. As mentioned in the introduction section, the occurrence of PF involves lung injury, the production of profibrotic factors, and the deposition of ECM. Reducing the expression of profibrotic factors and the production of ECM is extremely important for alleviating PF. Pirfenidone and nintedanib, approved by the FDA, have side effects and withdrawal symptoms [11, 13-14], and there is an urgent need to develop drugs with fewer side effects. TCM has accumulated valuable information alleviating lung diseases. WYJ, from steamed roots of Curcuma beauty, was included in the 2020 edition of the Pharmacopoeia of People's Republic of China. Modern studies have shown that the ingredients of WYJ can alleviate diseases through antibacterial, antitumor, anti-inflammatory, and antioxidant effects [16, 17]. Among the ingredients of WYJ, bisdemethoxycurcumin can reduce renal fibrosis through anti-inflammatory, antioxidant, and antiapoptotic effects [43, 44], ar-turmerone plays an antipsoriatic effect by inhibiting cell proliferation and reducing 
the expression of inflammatory cytokines [45], p-Methoxycinnamic exerts anticancer properties through anti-inflammatory effects [46], and curcumin has anti-pulmonary fibrosis effect in a murine model $[47,48]$. Based on the above research, we studied the impact of WYJ on PF and related molecular mechanisms.

It has developed many animal models to study the pathogenesis and treatment of different diseases in humans. The BLM-induced PF mouse model has been widely accepted as a model for studying PF. This model could cause a pulmonary pathological change, which is like that of patients with lung fibrosis $[49,50]$. Compared with the model group, we observed through pathological slices that WYJ treatment could decrease the infiltration of inflammatory cells and relieve thrombus and structure destruction of lung tissues (Figures $7(\mathrm{f})$ and $7(\mathrm{~g})$ ). Previous studies have indicated that TGF- $\beta 1, \alpha$-SMA, and ECM proteins (such as fibronectin, collagen I, and collagen III), and HYP are the main markers of the development of PF [7-10,51]. Our results showed that WYJ significantly inhibits the expression of these fibrosis markers in PF mice, which suggests that WYJ has an antifibrosis effect in the lungs. The weight change and survival curve also showed that WYJ can improve the quality of life of PF mice.

Various lung cell types (such as alveolar epithelial cells, endothelial cells, mesenchyme fibroblasts, and circulating fibroblasts) are involved in the occurrence and development of PF under pathological conditions [52]. However, differentiation of lung fibroblasts into myofibroblasts is essential for the development of PF $[37,38]$. As the most critical fibrosis-promoting factor, TGF- $\beta 1$ can induce lung fibroblasts to differentiate into myofibroblasts [7]. Therefore, we used TGF- $\beta 1$ to induce HPF and observed whether WYJ can inhibit HPF proliferation and differentiation. We found that WYJ significantly reduced the expression of fibrosis markers ( $\alpha$-SMA, collagen I) in HPF cells induced by TGF- $\beta 1$. These results are consistent with the results of the mouse model.

Multiple targets and multiple pathways regulate the occurrence of PF. So, only targeting one target for treatment will not eliminate the pathological activation of other targets in PF. Therefore, we used network pharmacology to screen out 9 critical targets of WYJ in the treatment of PF. MAPK1 (ERK2), MAPK8 (JNK1), and MAPK14 (p38- $\alpha$ ) are essential members of the MAPKs family. MAPKs signaling cascades regulate processes, including cell cycle progression, cell migration, cell survival, and differentiation [53]. More and more evidence showed that the MAPK pathway is involved in many aspects of $\mathrm{PF}$, such as the recruitment of fibroblasts and the deposition of extracellular matrix $[41,42,54,55]$. The production of IL- $1 \beta$ is achieved through a two-step process. The activation of the MAPK pathway participates in the synthesis of the inactive $31 \mathrm{kDa}$ precursor (pro-IL-1 $\beta$ ), and the inflammation induces the production of the active/mature form of IL- $1 \beta(17 \mathrm{kDa})$ $[56,57]$. IL- $1 \beta$ not only is an effective inducer of TGF- $\beta 1[58]$ but also causes BLM-induced pulmonary toxicity through the
caspase-1/IL-1 $\beta$ pathway [59]. EGFR is a transmembrane protein with intrinsic tyrosine kinase activity. Studies showed that EGFR is abnormally activated in animal models of PF and mediates the pathogenesis of pulmonary fibrosis [60-62]. Inhibition of EGFR reduced the phosphorylation of ERK1/2, leading to inhibition of the activation of profibrotic pathways [63]. KEGG analysis results showed that IL6, MAPK1, AKT1, EGFR, MAPK8, MAPK14, and IL1B were the key targets interacting closely with the MAPK signaling pathway. IL6, SRC, and ERGF can cause the activation of the downstream target STAT3, leading to lung fibrosis. STAT3 regulates cell growth, proliferation, differentiation, and migration [64]. Phosphorylated STAT3 has been reported to be involved in PF $[39,40,65,66]$. The primary receptor coupled with STAT3 is the gp130/IL-6 receptor family, including IL-6 [67, 68]. In the noncanonical TGF $\beta$ signaling, EGFR and SRC also mediate the phosphorylation and activation of STAT3 [69-72]. IL6, SRC, and ERGF can cause the activation of the downstream target STAT3, leading to lung fibrosis. To sum up, we speculated that WYJ might improve PF mainly via the MAPK and STAT3 signaling pathways. We performed experimental verification of the expression of essential proteins (MAPK1 and STAT3) in the two pathways. Western blot results showed that the phosphorylation of MAPK1 and STAT3 in the lung tissues of the model group was significantly increased, which is consistent with previous studies [18, 42, 63, 73]. WYJ alleviated PF by reducing the phosphorylation of MAPK1 and STAT3. In in vitro experiments, we also observed the same results, confirming the reliability of in vivo experiments.

The molecular docking results described above are helpful for a basic understanding of the mechanism of drug action. If the affinity energy is less than $-5 \mathrm{kcal} / \mathrm{mol}$, it indicates that the target has certain binding activity with the compound. We found that the affinity energies of most small molecules docking with proteins were less than $-5.0 \mathrm{kcal} / \mathrm{mol}$. We performed further analysis of the interaction between the target proteins and the compounds. The comparative analysis showed that positive control drug nintedanib showed good binding activity, but some of the active compounds of WYJ (bisdemethoxycurcumin, 1,2-dihydrocurcumin) showed higher binding activity with the core gene target to some extent, which also provides direction for the development of new drugs and subsequent research.

In conclusion, our study identified that WYJ alleviated pulmonary fibrosis. According to the results of network pharmacology and molecular docking, the pharmacological mechanism of WYJ in the treatment of PF was further validated by experiment. The inhibitory effect of WYJ on PF may function mainly by inhibiting MAPK and STAT3 signaling pathways, which provide new insights for the treatment of WYJ at PF. The method of exploring the pharmacological mechanism of WYJ used in our study provides a novel approach to explain the pharmacological basis of other herbs. Network pharmacology has limitations. More experiments are needed to verify the reliability antifibrotic effect of WYJ. 


\section{Data Availability}

The datasets used and analyzed during the current study are available from the corresponding author on reasonable request.

\section{Conflicts of Interest}

The authors declare that they have no conflicts of interest.

\section{Acknowledgments}

The manuscript is already published in the preprint https:// www.researchsquare.com/article/rs-923117/v1 [74]. No funding received.

\section{Supplementary Materials}

Supplementary Table S1: information about WYJ-related targets. Supplementary Table S2: information about PFrelated targets. Supplementary Table S3: targets information at the intersection of drug targets and disease targets. Supplementary Table S4: 48 key targets of WYJ against PF. Supplementary Table S5: core gene information filtered according to the "betweenness," "closeness," and "degree" values. Supplementary-Molecular Docking: information about molecular docking. (Supplementary Materials)

\section{References}

[1] B. Hochhegger, E. Marchiori, M. Zanon et al., "Imaging in idiopathic pulmonary fibrosis: diagnosis and mimics," Clinics, vol. 74, p. e225, 2019.

[2] K. C. Meyer, "Pulmonary fibrosis, part I: epidemiology, pathogenesis, and diagnosis," Expert Review of Respiratory Medicine, vol. 11, no. 5, pp. 343-359, 2017.

[3] M. Selman and A. Pardo, "Revealing the pathogenic and aging-related mechanisms of the enigmatic idiopathic pulmonary fibrosis. an integral model," American Journal of Respiratory and Critical Care Medicine, vol. 189, no. 10, pp. 1161-1172, 2014.

[4] F. J. Martinez, H. R. Collard, A. Pardo et al., "Idiopathic pulmonary fibrosis," Nature Reviews. Disease Primers, vol. 3, p. 17074, 2017.

[5] S. J. Cho and H. W. Stout-Delgado, "Aging and lung disease," Annual Review of Physiology, vol. 82, pp. 433-459, 2020.

[6] M. J. Abramson, T. Murambadoro, S. M. Alif et al., "Occupational and environmental risk factors for idiopathic pulmonary fibrosis in Australia: case-control study," Thorax, vol. 75, no. 10, pp. 864-869, 2020.

[7] D. Chanda, E. Otoupalova, S. R. Smith, T. Volckaert, S. P. De Langhe, and V. J. Thannickal, "Developmental pathways in the pathogenesis of lung fibrosis," Molecular Aspects of Medicine, vol. 65, pp. 56-69, 2019.

[8] A. Leask and D. J. Abraham, "TGF-beta signaling and the fibrotic response," The FASEB Journal: Official Publication of the Federation of American Societies for Experimental Biology, vol. 18, no. 7, pp. 816-827, 2004.

[9] L. Richeldi, H. R. Collard, and M. G. Jones, "Idiopathic pulmonary fibrosis," Lancet, vol. 389, no. 10082, pp. 1941-1952, 2017.
[10] D. J. Lederer and F. J. Martinez, "Idiopathic pulmonary fibrosis," New England Journal of Medicine, vol. 378, no. 19, pp. 1811-1823, 2018.

[11] T. Ogura and H. Kitamura, "Tolerability of treatment with pirfenidone or nintedanib for pulmonary fibrosis in the real world," Respirology, vol. 22, no. 6, pp. 1051-1052, 2017.

[12] A. Azuma, T. Nukiwa, E. Tsuboi et al., "Double-blind, placebo-controlled trial of pirfenidone in patients with idiopathic pulmonary fibrosis," American Journal of Respiratory and Critical Care Medicine, vol. 171, no. 9, pp. 1040-1047, 2005.

[13] K. R. Flaherty, A. U. Wells, V. Cottin et al., "Nintedanib in progressive fibrosing interstitial lung diseases," New England Journal of Medicine, vol. 381, no. 18, pp. 1718-1727, 2019.

[14] J. A. Rodríguez-Portal, "Efficacy and safety of nintedanib for the treatment of idiopathic pulmonary fibrosis: an update," Drugs in $R$ \& $D$, vol. 18, no. 1, pp. 19-25, 2018.

[15] L. C. Li and L. D. Kan, "Traditional Chinese medicine for pulmonary fibrosis therapy: progress and future prospects," Journal of Ethnopharmacology, vol. 198, pp. 45-63, 2017.

[16] Y. Li, Y. Wu, Y. Li, and F. Guo, "Review of the traditional uses, phytochemistry, and pharmacology of Curcuma wenyujin Y. H. Chen et C. Ling," Journal of Ethnopharmacology, vol. 269, Article ID 113689, 2021.

[17] G. P. Yin, Q. Z. Zhang, Y. W. An, J. J. Zhu, and Z. M. Wang, "[Advance in chemical constituents and pharmacological activity of Curcuma wenyujin]," Zhongguo Zhong yao za zhi, vol. 37, no. 22, pp. 3354-3360, 2012.

[18] H. Xie, D. Su, J. Zhang et al., "Raw and vinegar processed Curcuma wenyujin regulates hepatic fibrosis via bloking TGF$\beta /$ Smad signaling pathways and up-regulation of MMP-2/ TIMP-1 ratio," Journal of Ethnopharmacology, vol. 246, Article ID 111768, 2020.

[19] R. Singh, V. K. Bhardwaj, J. Sharma, P. Das, and R. Purohit, "Identification of selective cyclin-dependent kinase 2 inhibitor from the library of pyrrolone-fused benzosuberene compounds: an in silico exploration," Journal of Biomolecular Structure \& Dynamics, pp. 1-9, 2021.

[20] R. Singh, V. K. Bhardwaj, J. Sharma, P. Das, and R. Purohit, "Discovery and in silico evaluation of aminoarylbenzosuberene molecules as novel checkpoint kinase 1 inhibitor determinants," Genomics, vol. 113, no. 1 Pt 2, pp. 707-715, 2021.

[21] A. L. Hopkins, "Network pharmacology: the next paradigm in drug discovery," Nature Chemical Biology, vol. 4, no. 11, pp. 682-690, 2008.

[22] W. Wang, T. Liu, L. Yang et al., "Study on the multi-targets mechanism of triphala on cardio-cerebral vascular diseases based on network pharmacology," Biomedicine \& pharmacotherapy, vol. 116, Article ID 108994, 2019.

[23] G. Yu, Z. Luo, Y. Zhou et al., "Uncovering the pharmacological mechanism of Carthamus tinctorius L. on cardiovascular disease by a systems pharmacology approach," Biomedicine \& Pharmacotherapy, vol. 117, Article ID 109094, 2019.

[24] H. Qin, H. Chen, Y. Zou et al., "Systematic investigation of the mechanism of Cichorium glandulosum on type 2 diabetes mellitus accompanied with non-alcoholic fatty liver rats," Food \& Function, vol. 10, no. 5, pp. 2450-2460, 2019.

[25] K. F. Taha, M. Khalil, M. S. Abubakr, and E. Shawky, "Identifying cancer-related molecular targets of Nandina domestica Thunb. by network pharmacology-based analysis in combination with chemical profiling and molecular 
docking studies," Journal of Ethnopharmacology, vol. 249, Article ID 112413, 2020.

[26] J. Sharma, V. K. Bhardwaj, P. Das, and R. Purohit, "Identification of naturally originated molecules as $\gamma$-aminobutyric acid receptor antagonist," Journal of Biomolecular Structure \& Dynamics, vol. 39, no. 3, pp. 911-922, 2021.

[27] V. Rajendran, C. Gopalakrishnan, and R. Sethumadhavan, "Pathological role of a point mutation (T315I) in BCR-ABL1 protein-A computational insight," Journal of Cellular Biochemistry, vol. 119, no. 1, pp. 918-925, 2018.

[28] A. Daina, O. Michielin, and V. Zoete, "SwissTargetPrediction: updated data and new features for efficient prediction of protein targets of small molecules," Nucleic Acids Research, vol. 47, no. W1, pp. W357-w364, 2019.

[29] G. Stelzer, N. Rosen, I. Plaschkes et al., "The GeneCards suite: from gene data mining to disease genome sequence analyses," Current Protocols in Bioinformatics, vol. 54, pp. 1.30.1-1.30.33, 2016.

[30] J. S. Amberger, C. A. Bocchini, F. Schiettecatte, A. F. Scott, and A. Hamosh, "OMIM.org: online Mendelian inheritance in Man $\left(\mathrm{OMIM}^{\circledR}\right)$, an online catalog of human genes and genetic disorders," Nucleic Acids Research, vol. 43, pp. D789-D798, 2015.

[31] D. Szklarczyk, A. L. Gable, K. C. Nastou et al., "The STRING database in 2021: customizable protein-protein networks, and functional characterization of user-uploaded gene/measurement sets," Nucleic Acids Research, vol. 49, no. D1, pp. D605-d612, 2021.

[32] G. Dennis Jr., B. T. Sherman, D. A. Hosack et al., "DAVID: database for annotation, visualization, and integrated discovery," Genome Biology, vol. 4, no. 5, p. P3, 2003.

[33] W. Huang da, B. T. Sherman, and R. A. Lempicki, "Bioinformatics enrichment tools: paths toward the comprehensive functional analysis of large gene lists," Nucleic Acids Research, vol. 37, no. 1, pp. 1-13, 2009.

[34] K. Ask, P. Bonniaud, K. Maass et al., "Progressive pulmonary fibrosis is mediated by TGF-beta isoform 1 but not TGFbeta3," The International Journal of Biochemistry \& Cell Biology, vol. 40, no. 3, pp. 484-495, 2008.

[35] V. J. Thannickal, D. Y. Lee, E. S. White et al., "Myofibroblast differentiation by transforming growth factor-betal is dependent on cell adhesion and integrin signaling via focal adhesion kinase," Journal of Biological Chemistry, vol. 278, no. 14 , pp. 12384-12389, 2003.

[36] A. Pardo and M. Selman, "Matrix metalloproteases in aberrant fibrotic tissue remodeling," Proceedings of the American Thoracic Society, vol. 3, no. 4, pp. 383-388, 2006.

[37] N. Xie, Z. Tan, S. Banerjee et al., "Glycolytic reprogramming in myofibroblast differentiation and lung fibrosis," American Journal of Respiratory and Critical Care Medicine, vol. 192, no. 12, pp. 1462-1474, 2015.

[38] S. T. Lehtonen, A. Veijola, H. Karvonen et al., "Pirfenidone and nintedanib modulate properties of fibroblasts and myofibroblasts in idiopathic pulmonary fibrosis," Respiratory Research, vol. 17, p. 14, 2016.

[39] T. T. Le, H. Karmouty-Quintana, E. Melicoff et al., "Blockade of IL-6 Trans signaling attenuates pulmonary fibrosis," Journal of Immunology: 1950), vol. 193, no. 7, pp. 3755-3768, 2014.

[40] Y. P. Moodley, A. K. Scaffidi, N. L. Misso et al., "Fibroblasts isolated from normal lungs and those with idiopathic pulmonary fibrosis differ in interleukin-6/gp130-mediated cell signaling and proliferation," American Journal Of Pathology, vol. 163 , no. 1 , pp. $345-354,2003$.

[41] K. M. Antoniou, G. A. Margaritopoulos, G. Soufla et al., "Expression analysis of Akt and MAPK signaling pathways in lung tissue of patients with idiopathic pulmonary fibrosis (IPF)," Journal of Receptors and Signal Transduction Research, vol. 30, no. 4, pp. 262-269, 2010.

[42] K. Yoshida, K. Kuwano, N. Hagimoto et al., "MAP kinase activation and apoptosis in lung tissues from patients with idiopathic pulmonary fibrosis," The Journal of Pathology, vol. 198, no. 3, pp. 388-396, 2002.

[43] F. Jin, X. Chen, H. Yan et al., "Bisdemethoxycurcumin attenuates cisplatin-induced renal injury through anti-apoptosis, anti-oxidant and anti-inflammatory," European Journal of Pharmacology, vol. 874, Article ID 173026, 2020.

[44] F. Jin, Y. Jin, J. Du et al., "Bisdemethoxycurcumin protects against renal fibrosis via activation of fibroblast apoptosis," European Journal of Pharmacology, vol. 847, pp. 26-31, 2019.

[45] S. Yang, J. Liu, J. Jiao, and L. Jiao, "Ar-turmerone exerts antiproliferative and anti-inflammatory activities in $\mathrm{HaCaT}$ keratinocytes by inactivating hedgehog pathway," Inflammation, vol. 43, no. 2, pp. 478-486, 2020.

[46] R. T. T. Paim, P. S. A. Rodrigues, J. Silva et al., "p-Methoxycinnamic acid diesters lower dyslipidemia, liver oxidative stress and toxicity in high-fat diet fed mice and human peripheral blood lymphocytes," Nutrients, vol. 12, no. 1, 2020.

[47] J. C. Lee, P. A. Kinniry, E. Arguiri et al., "Dietary curcumin increases antioxidant defenses in lung, ameliorates radiationinduced pulmonary fibrosis, and improves survival in mice," Radiation Research, vol. 173, no. 5, pp. 590-601, 2010.

[48] Y. J. Cho, C. O. Yi, B. T. Jeon et al., "Curcumin attenuates radiation-induced inflammation and fibrosis in rat lungs," Korean Journal of Physiology and Pharmacology, vol. 17, no. 4, pp. 267-274, 2013.

[49] W. A. Border and N. A. Noble, "Transforming growth factor beta in tissue fibrosis," New England Journal of Medicine, vol. 331, no. 19, pp. 1286-1292, 1994.

[50] V. Della Latta, A. Cecchettini, S. Del Ry, and M. A. Morales, "Bleomycin in the setting of lung fibrosis induction: from biological mechanisms to counteractions," Pharmacological Research, vol. 97, pp. 122-130, 2015.

[51] X. Yang, L. Wu, G. Li, Q. Ran, and L. Zhang, "[Alphacalcidol combined with dexamethasone for reducing pulmonary fibrosis in mice and its mechanism],"Xi Bao Yu Fen Zi Mian Yi Xue Za Zhi, vol. 33, no. 4, pp. 488-491, 2017.

[52] R. M. Strieter and B. Mehrad, "New mechanisms of pulmonary fibrosis," Chest, vol. 136, no. 5, pp. 1364-1370, 2009.

[53] D. K. Morrison, "MAP kinase pathways," Cold Spring Harbor Perspectives in Biology, vol. 4, no. 11, 2012.

[54] J. F. Alcorn, J. van der Velden, A. L. Brown, B. McElhinney, C. G. Irvin, and Y. M. Janssen-Heininger, "c-Jun N-terminal kinase 1 is required for the development of pulmonary fibrosis," American Journal of Respiratory Cell and Molecular Biology, vol. 40, no. 4, pp. 422-432, 2009.

[55] I. Kolosova, D. Nethery, and J. A. Kern, "Role of Smad $2 / 3$ and p38 MAP kinase in TGF- $\beta 1$-induced epithelial-mesenchymal transition of pulmonary epithelial cells," Journal of Cellular Physiology, vol. 226, no. 5, pp. 1248-1254, 2011.

[56] L. Franchi, T. Eigenbrod, R. Muñoz-Planillo, and G. Nuñez, "The inflammasome: a caspase-1-activation platform that regulates immune responses and disease pathogenesis," $\mathrm{Na}$ ture Immunology, vol. 10, no. 3, pp. 241-247, 2009.

[57] S. Lee, G. Y. Suh, S. W. Ryter, and A. M. Choi, "Regulation and function of the nucleotide binding domain leucine-rich repeatcontaining receptor, pyrin domain-containing-3 inflammasome in lung disease," American Journal of Respiratory Cell and Molecular Biology, vol. 54, no. 2, pp. 151-160, 2016. 
[58] M. Kolb, P. J. Margetts, D. C. Anthony, F. Pitossi, and J. Gauldie, "Transient expression of IL-1beta induces acute lung injury and chronic repair leading to pulmonary fibrosis," Journal of Clinical Investigation, vol. 107, no. 12, pp. 1529-1536, 2001.

[59] P. Gasse, C. Mary, I. Guenon et al., "IL-1R1/MyD88 signaling and the inflammasome are essential in pulmonary inflammation and fibrosis in mice," Journal of Clinical Investigation, vol. 117, no. 12, pp. 3786-3799, 2007.

[60] Y. Zhou, J. Y. Lee, C. M. Lee et al., “Amphiregulin, an epidermal growth factor receptor ligand, plays an essential role in the pathogenesis of transforming growth factor- $\beta$-induced pulmonary fibrosis," Journal of Biological Chemistry, vol. 287, no. 50, pp. 41991-42000, 2012.

[61] S. J. DiCamillo, S. Yang, M. V. Panchenko et al., "Neutrophil elastase-initiated EGFR/MEK/ERK signaling counteracts stabilizing effect of autocrine TGF-beta on tropoelastin mRNA in lung fibroblasts," American Journal of Physiology. Lung Cellular and Molecular Physiology, vol. 291, no. 2, pp. L232-L243, 2006.

[62] Y. Ishii, S. Fujimoto, and T. Fukuda, "Gefitinib prevents bleomycin-induced lung fibrosis in mice," American Journal of Respiratory and Critical Care Medicine, vol. 174, no. 5, pp. 550-556, 2006.

[63] L. Sheng, M. Yang, W. Ding et al., "Epidermal growth factor receptor signaling mediates aldosterone-induced profibrotic responses in kidney," Experimental Cell Research, vol. 346, no. 1, pp. 99-110, 2016.

[64] D. E. Levy and J. E. Darnell Jr., "Stats: transcriptional control and biological impact, Nature reviews," Molecular Cell Biology, vol. 3, no. 9, pp. 651-662, 2002.

[65] D. V. Pechkovsky, C. M. Prêle, J. Wong et al., "STAT3-mediated signaling dysregulates lung fibroblast-myofibroblast activation and differentiation in UIP/IPF," American Journal Of Pathology, vol. 180, no. 4, pp. 1398-1412, 2012.

[66] R. J. O’Donoghue, D. A. Knight, C. D. Richards et al., "Genetic partitioning of interleukin-6 signalling in mice dissociates Stat3 from Smad3-mediated lung fibrosis," EMBO Molecular Medicine, vol. 4, no. 9, pp. 939-951, 2012.

[67] S. Rose-John, "Interleukin-6 family cytokines," Cold Spring Harbor Perspectives in Biology, vol. 10, no. 2, 2018.

[68] M. Mihara, M. Hashizume, H. Yoshida, M. Suzuki, and M. Shiina, "IL-6/IL-6 receptor system and its role in physiological and pathological conditions," Clinical Science: 1979), vol. 122, no. 4, pp. 143-159, 2012.

[69] P. O. Hackel, E. Zwick, N. Prenzel, and A. Ullrich, "Epidermal growth factor receptors: critical mediators of multiple receptor pathways," Current Opinion in Cell Biology, vol. 11, no. 2, pp. 184-189, 1999.

[70] S. J. Forrester, T. Kawai, S. O’Brien, W. Thomas, R. C. Harris, and S. Eguchi, "Epidermal growth factor receptor transactivation: mechanisms, pathophysiology, and potential therapies in the cardiovascular system," Annual Review of Pharmacology and Toxicology, vol. 56, pp. 627-653, 2016.

[71] A. Akhmetshina, C. Dees, M. Pileckyte et al., "Dual inhibition of c-abl and PDGF receptor signaling by dasatinib and nilotinib for the treatment of dermal fibrosis," The FASEB Journal: Official Publication of the Federation of American Societies for Experimental Biology, vol. 22, no. 7, pp. 2214-2222, 2008.

[72] Y. Liu, H. Liu, C. Meyer et al., "Transforming growth factor- $\beta$ (TGF- $\beta$ )-mediated connective tissue growth factor (CTGF) expression in hepatic stellate cells requires Stat3 signaling activation," Journal of Biological Chemistry, vol. 288, no. 42, pp. 30708-30719, 2013.
[73] Y. Liu, Y. Wang, F. Lu, L. Wang, L. Miao, and X. Wang, "BTB and $\mathrm{CNC}$ homology 1 inhibition ameliorates fibrosis and inflammation via blocking ERK pathway in pulmonary fibrosis," Experimental Lung Research, vol. 47, no. 2, pp. 67-77, 2021.

[74] L. Wang, W. X. Zhu, and R. Sun, "Network pharmacology prediction and molecular docking-based strategy to discover the potential pharmacological mechanism of Wen-yu-jin against pulmonary fibrosis in mouse model," 2021. 